\title{
Estimation of the Parameters of Type-II Discrete Weibull Distribution Under Type-I Censoring
}

\author{
Mohamed S. A. Muiftah ${ }^{1} \&$ Samir K. Ashour ${ }^{2}$ \\ ${ }^{1}$ Department of Statistics, Faculty of Science, University of Benghazi, Benghazi, Libya \\ ${ }^{2}$ Department of Mathematical Statistics, FSSR, Cairo University, Cairo, Egypt \\ Correspondence: Mohamed S. A. Muiftah, Department of Statistics, Faculty of Science, University of Benghazi, \\ Benghazi, Libya
}

Received: May 29, 2021 Accepted: July 12021 Online Published: August 15, 2021

doi:10.5539/ijsp.v10n5p1 URL: https://doi.org/10.5539/ijsp.v10n5p1

\begin{abstract}
Maximum likelihood and proportion estimators of the parameters of the discrete Weibull type II distribution with type I censored data are discussed. A simulation study is performed to generate data from this distribution for suggested values of its parameters and to get the Maximum likelihood estimates of the parameters numerically. The method of proportions suggested by Khan et al. (1989) is also used to estimate the model's parameters. Numerical examples are used to perform a comparison study between the two method results according the values of the estimates and their corresponding mean squared errors.
\end{abstract}

Keywords: discrete Weibull distribution, Maximum likelihood estimates, method of proportions, type-I censoring

\section{Introduction}

Discrete versions of some continuous distributions were suggested by some researchers using different discretizing methods. These methods and distributions were very good reviewed by Chakraborty (2015) and extensively studied by Muiftah (2018). In this paper, the maximum likelihood (ML) and proportion estimators of the parameters of the discrete Weibull type II [DW(II)] distribution with type I censored data are discussed. The derivation of the first and second types of the discrete Weibull distribution is introduced firstly. Methods used to estimate the distribution parameters are then introduced. A simulation study is performed to generate data from the suggested distribution and to get the ML estimates of the parameters. The proportion estimates are also calculated. The results are compared. Tables and graphs are used to illustrate the distribution and the results in a good manner. Finally the dissection and conclusion are given.

\section{Derivation of the Discrete Weibull Distribution}

The first discrete version of the Weibull distribution DW(I) was introduced by Nakagawa and Osaki (1975) as follows:

If $\mathrm{T}$ is a random variable following the continuous Weibull distribution with a shape parameter $\alpha>0$ and a scale parameter $\theta>0[T \sim W(\alpha, \theta)]$, then the probability density function (pdf), the cumulative distribution function (cdf), the survival function (sf), and the failure rate (fr) of $\mathrm{T}$ are respectively:

$$
\begin{gathered}
f_{T}(t)=\alpha \theta t^{\alpha-1} e^{-\theta t^{\alpha}}, t>0 \\
F_{T}(t)=P(T \leq t)=1-e^{-\theta t^{\alpha}}, \\
S_{T}(t)=e^{-\theta t^{\alpha}}, \\
\text { and } \quad h_{T}(t)=\alpha \theta t^{\alpha-1},
\end{gathered}
$$

then, by inserting the sf given by (1) in the discretization formula:

$$
P(Y=y)=S_{T}(y)-S_{T}(y+1)
$$

the pmf of the DW(I) distribution will be given by: 


$$
P(Y=y)=e^{-\theta y^{\alpha}}-e^{-\theta(y+1)^{\alpha}}, \quad y=0,1, \cdots
$$

By substituting $e^{-\theta}=q$, the probability mass function (pmf), sf, and fr of the DW(I) are respectively:

$$
\begin{aligned}
P(Y=y) & =P_{y}=q^{y^{\alpha}}-q^{(y+1)^{\alpha}}, \quad 0<q<1, \quad y=0,1, \cdots \\
S_{Y}(y) & =e^{-\theta y^{\alpha}}=q^{y^{\alpha}}, \\
h_{Y}(q, \alpha) & =\frac{P_{y}}{\sum_{i=y}^{\infty} P_{i}}=1-q^{(y+1)^{\alpha}-y^{\alpha}} .
\end{aligned}
$$

The first two moments of the DW(I) distribution are given by:

$$
E(Y)=\sum_{y=0}^{\infty} q^{y^{\alpha}}, \quad \text { and } \quad E\left(Y^{2}\right)=2 \sum_{y=0}^{\infty} y q^{y^{\alpha}}+E(Y) \text {. }
$$

Khan et al. (1989) compared between the estimates of the parameters q and $\alpha$ obtained by method of moments and method of proportions. Kulasecara (1994) discussed the approximate ML estimation of the parameters under right censoring and compared his estimators with the proportion estimators introduced by Khan et al. (1989).

Stein and Dattero (1984) introduced their discrete analogue of the Weibull distribution [DW(II)] by inserting the fr given by (2) in the discretization formula:

$$
\begin{aligned}
P(Y=y) & =\left[1-h_{T}(0)\right]\left[1-h_{T}(1)\right] \cdots\left[1-h_{T}(y-1)\right]\left\{1-\left[1-h_{T}(y)\right]\right\} \\
& =\left[1-h_{T}(0)\right]\left[1-h_{T}(1)\right] \cdots\left[1-h_{T}(y-1)\right] h_{T}(y) \\
& =\left\{\begin{array}{cc}
h_{T}(0), & y=0 \\
{\left[1-h_{T}(0)\right]\left[1-h_{T}(1)\right] \cdots\left[1-h_{T}(y-1)\right] h_{T}(y), \quad y=1,2, \cdots, d} & \text { otherwise }
\end{array}\right.
\end{aligned}
$$

and defined it by the fr: $\quad h_{Y}(y)=\left\{\begin{array}{ll}C y^{\alpha-1}, & y=1,2, \cdots, d \\ 0, & \text { otherwise }\end{array}\right.$,

where, $\quad 0<C=\alpha \theta \leq 1$, and the parameter $d$ is determined in such a way that $0 \leq h_{T}(t)<1$,

where, with $\alpha>0$ and $0<C \leq d, \quad d=\left\{\begin{array}{cc}\text { int }\left\{C^{-(\alpha-1)^{-1}}\right. & \}^{+}, \quad \alpha>1 \\ +\infty, & \alpha \leq 1\end{array}\right.$,

where, int $\{.\}^{+}$represents the integer part of the quantity inside the braces.

Khan et al. (1989) presented the pmf of the DW(II) distribution to be:

$$
\begin{aligned}
P(Y=y) & =S_{Y}(y-1) h_{Y}(y) \\
& =\left[1-h_{T}(1)\right]\left[1-h_{T}(2)\right] \cdots\left[1-h_{T}(y-1)\right] h_{T}(y) \\
& =C y^{\alpha-1} \prod_{i=1}^{y-1}\left(1-C i^{\alpha-1}\right), \quad y=1,2, \cdots, d
\end{aligned}
$$


Chakraborty (2015) gave the sf of this distribution in the form:

$$
S_{Y}(y)=\prod_{i=1}^{y-1}\left(1-C i^{\alpha-1}\right), \quad y=1,2, \cdots, d
$$

The first two moments of the DW(II) distribution are:

$$
\mathrm{E}(\mathrm{Y})=\sum_{\mathrm{y}=1}^{\mathrm{d}} \mathrm{Cy} \mathrm{y}^{\alpha} \prod_{\mathrm{i}=1}^{\mathrm{y}-1}\left(1-\mathrm{Ci}^{\alpha-1}\right), \quad \text { and } E\left(Y^{2}\right)=\sum_{y=1}^{d} C y^{\alpha+1} \prod_{i=1}^{y-1}\left(1-C i^{\alpha-1}\right) .
$$

The DW(II) distribution reduces to the geometric distribution for $\mathrm{C}=1-\mathrm{q}$ and $\alpha=1$.

\section{Estimation of the Parameters}

\subsection{The Maximum Likelihood Estimation}

Let $Y_{1}, Y_{2}, \ldots, Y_{n}$ be $n$ identically independently distributed (i.i.d.) observations from the DW(II) distribution given by (2.3) each is right censored by a fixed termination time $\gamma$. Suppose that $r$ observations from this sample are less than $\gamma$ and $(n-r)$ observations are greater than $\gamma$, then the likelihood function of these observations will be:

$$
\begin{aligned}
L & =\frac{n !}{(n-r) !} \prod_{j=1}^{r}\left\{\left[P\left(Y=y_{j}\right)\right]\left[S_{Y}(y)\right]^{(n-r)}\right\} \\
& =\frac{n !}{(n-r) !} \prod_{j=1}^{r}\left\{\left[\alpha \theta y_{j}^{\alpha-1} \prod_{i=1}^{y_{j}-1}\left(1-\alpha \theta i^{\alpha-1}\right)\right]\left[\prod_{i=1}^{y_{j}-1}\left(1-\alpha \theta i^{\alpha-1}\right)\right]^{(n-r)}\right\}
\end{aligned}
$$

The log-likelihood function is then given by:

$$
\begin{aligned}
\log L & =\log \left[\frac{n !}{(n-r) !}\right]+\log \prod_{j=1}^{r}\left\{\left[\alpha \theta y_{j}^{\alpha-1} \prod_{i=1}^{y_{j}-1}\left(1-\alpha \theta i^{\alpha-1}\right)\right]\left[\prod_{i=1}^{y_{j}-1}\left(1-\alpha \theta i^{\alpha-1}\right)\right]^{(n-r)}\right\} \\
& =\log \left[\frac{n !}{(n-r) !}\right]+r \log \alpha+r \log \theta+(\alpha-1) \sum_{j=1}^{r} \log y_{j}+(n-r+1) \sum_{j=1}^{r} \sum_{i=1}^{y_{j}-1} \log \left(1-\alpha \theta i^{\alpha-1}\right)
\end{aligned}
$$

The first partial derivatives of $\log L$ w.r.t $\alpha$ and $\theta$ are respectively:

and

$$
\begin{aligned}
& \frac{\partial \log L}{\partial \alpha}=\frac{r}{\alpha}+\sum_{j=1}^{r} \log y_{j}-(n-r+1) \sum_{j=1}^{r} \sum_{i=1}^{y_{j}-1}\left(\frac{\theta i^{\alpha-1}(\alpha \log i+1)}{1-\alpha \theta i^{\alpha-1}}\right), \\
& \frac{\partial \log L}{\partial \theta}=\frac{r}{\theta}-(n-r+1) \sum_{j=1}^{r} \sum_{i=1}^{y_{j}-1}\left(\frac{\alpha i^{\alpha-1}}{1-\alpha \theta i^{\alpha-1}}\right)
\end{aligned}
$$

which will be numerically maximized to get the ML estimates of $\theta$ and $\alpha$. 


\subsection{Estimation Using the Method of Proportions}

In the DW(II) model $P(Y=1)=P_{1}=C$ may be estimated through the proportion of ones in the sample, $\frac{f_{1}}{n}$, where, $f_{1}$ is the number of ones in the sample, and $n$ is the sample size, then an estimate of $C$ is given by: $\hat{C}=\hat{P}_{l}=\frac{f_{l}}{n}$.

Similarly, $P(Y=2)=P_{2}=C 2^{\alpha-1}(1-C)$ may be estimated through the proportion of 2 's in the sample, $\frac{f_{2}}{n}$, where, $f_{2}$ is the number of 2's in the sample, then:

$$
\begin{aligned}
\hat{P}_{2}=\hat{C} 2^{\hat{\alpha}-1}(1-\hat{C}) & \Rightarrow 2^{\hat{\alpha}-1}=\frac{\hat{P}_{2}}{\hat{C}(1-\hat{C})} \Rightarrow(\hat{\alpha}-1) \log 2=\log \left[\frac{\hat{P}_{2}}{\hat{C}(1-\hat{C})}\right] \\
& \Rightarrow \hat{\alpha} \log 2=\log \left[\frac{\hat{P}_{2}}{\hat{C}(1-\hat{C})}\right]+\log 2
\end{aligned}
$$

Substituting for $\hat{C}$ and $\hat{P}_{2}$, then an estimator of $\alpha$ is given by:

$$
\hat{\alpha}_{p}=(\log 2)^{-1}\left[\log \left(\frac{\frac{f_{2}}{n}}{\frac{f_{1}}{n}\left(1-\frac{f_{1}}{n}\right)}\right)+\log 2\right]
$$

then, the estimator of $\theta$ is given by: $\hat{\theta}_{p}=\frac{\hat{C}}{\hat{\alpha}_{p}}=\frac{\left(\frac{f_{1}}{n}\right)}{\hat{\alpha}_{p}}$.

The cdfs $\hat{F}_{Y}(1)=\hat{P}(Y>1)=1-\hat{C}=1-\frac{f_{1}}{n} \quad$ and $\quad \hat{F}_{Y}(2)=\hat{P}(Y>2)=1-\frac{f_{1}}{n}-\frac{f_{2}}{n}$ are empirical cdfs and are unbiased and consistent estimators of the actual cdfs $F_{Y}(1)$ and $F_{Y}(2)$ respectively. [Khan et al. (1989)], then, replacing $\frac{f_{1}}{n}$ by $\left[1-\hat{F}_{Y}(1)\right]$ and $\frac{f_{2}}{n}$ by $\left[\hat{F}_{Y}(2)-\bar{F}_{Y}(1)\right]$, equations (3.1) and (3.2) may be rewritten as:

$$
\hat{\alpha}_{p}=(\log 2)^{-1}\left[\log \left(\frac{\hat{F}_{Y}(2)-\hat{F}_{Y}(1)}{\hat{F}_{Y}(1)\left[1-\hat{F}_{Y}(1)\right]}\right)+\log 2\right], \quad \hat{\theta}_{p}=\frac{1-\hat{F}_{Y}(1)}{\hat{\alpha}_{p}}
$$

Since the empirical probabilities are used to estimate $F_{Y}(1)=P(Y>1)$ and $F_{Y}(2)=P(Y>2)$, then, $\left(1-\frac{f_{1}}{n}\right)$ and $\left(1-\frac{f_{1}}{n}-\frac{f_{2}}{n}\right)$ are unbiased and consistent estimators for $F_{Y}(1)$ and $F_{Y}(2)$ respectively, consequently, $\hat{\alpha}_{p}$ and $\hat{\theta}_{p}$ are also unbiased and consistent estimators of $\alpha$ and $\theta$ respectively.

\section{The Simulation Study (Numerical Examples)}

Different values for $\alpha$ and $\beta$ were suggested such that $0<\alpha \beta<1$ and $\mathrm{d}$ is finite (the infinite case is not considered in our study), i.e. $(\alpha>1, \beta<1)$, and a Mathcad program is used to simulate data from the suggested distribution and to obtain the ML estimates using 1000 replications. A sample of 150 samples is randomly chosen from the simulated data of each DW(II) distribution used in the ML estimation, a Microsoft Excel program is used to calculate the number of 1's and 
2's in each chosen sample and to obtain the proportion estimates $\breve{\alpha}_{i}$ and $\breve{\beta}_{p i}(i=1, \ldots, 150)$ from each sample, then the mean of the sample estimates is used to be the proportion estimate of the distribution, i.e. $\breve{\alpha}_{p}=\frac{\sum_{i=1}^{150} \breve{\alpha}_{p_{i}}}{150}$ and $\breve{\beta}=\frac{\sum_{i=1}^{150} \breve{\beta}_{i}}{150}$.

In the case of $n=15$, some selected samples are found not to contain 1's or 2's, hence no proportion estimates are available, so these samples were excluded and replaced by other randomly selected samples.

The ML and the proportion estimates for the parameters $\alpha$ and $\beta$ from DW(II) $(\alpha, \beta)$ are obtained for different $\gamma$ which are found to be equivalent to the values of $r(r=80 \% n, 90 \% n, 95 \% n, 98 \% n$, and $100 \% n)$ for each $n=15,25,50,100$, and 150 .

4.1 For $(\alpha=1.8, \beta=0.1, d=9)$

The pmf of DW(II) $(1.8,0.1)$ is given by Table $4.1 \mathrm{a}$ and the bar chart given by Figure 4.1.

Table 4.1a. The pmf of DW(II) $(1.8,0.1)$ distribution

\begin{tabular}{|c|c|c|c|c|c|}
\hline $\mathrm{y}$ & 1 & 2 & 3 & 4 & 5 \\
\hline $\mathrm{P}(\mathrm{y})$ & 0.18 & 0.256987 & 0.244055 & 0.174042 & 0.094529 \\
\hline $\mathrm{y}$ & 6 & 7 & 8 & 9 & $\Sigma$ \\
\hline $\mathrm{P}(\mathrm{y})$ & 0.038029 & 0.010551 & 0.001717 & $9.42 \times 10^{-5}$ & 1.000004 \\
\hline
\end{tabular}

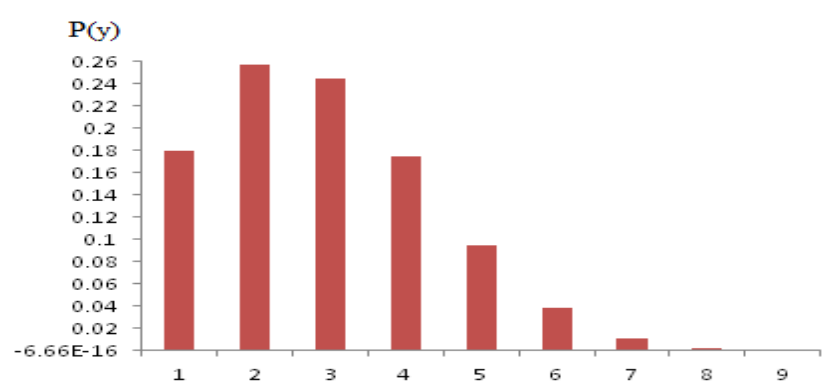

Figure 4.1 The pmf of DW(II) $(1.8,0.1)$

The first two moments and the variance of DW(II) $(1.8,0.1)$ are respectively given by:

$$
\mathrm{E}(\mathrm{Y})=2.912, \mathrm{E}\left(\mathrm{Y}^{2}\right)=10.556 \text {, and } \operatorname{Var}(\mathrm{Y})=2.076 \text {. }
$$

The ML and the proportion estimates for the parameter $\alpha$ from $\operatorname{DW}(\mathrm{II})(1.8,0.1)$ for different $\gamma(\gamma=4,5,6,7$, and 9) which are found to be respectively equivalent to the values of $r(r=80 \% n, 90 \% n, 95 \% n, 98 \% n$, and $100 \% n)$ for each sample size and the corresponding MSEs are given in table (4.1b) which constitutes of six columns, the first column contains the sample size $n(n=15,25,50,100$, and 150), the second column contains $\mathrm{r}$ (the number of observations less than $\gamma$ ) for each $n$, the third column contains the ML estimate of $\alpha$, the fourth column contains the MSE of the ML estimate of $\alpha$, the fifth column contains the proportion estimate of $\alpha$, and the sixth column contains the MSE of the proportion estimate of $\alpha$. The estimates of the parameter $\beta$ from DW(II) $(1.8,0.1)$ for different $\gamma$ and the corresponding MSEs are given in Table 4.1c which constitutes of the same contents of Table $4.1 \mathrm{~b}$ considering $\beta$ instead of $\alpha$.

The estimates of the parameter $\alpha$ from DW(II) $(1.8,0.1)$ for different $\mathrm{n}$ and the corresponding MSEs are given in Table $4.1 \mathrm{~d}$ which constitutes of seven columns, the first column contains the percentage $\%$ from n $(80 \%, 90 \%, 95 \%$, 98\%, and 100\%), the second column contains the termination time $\gamma(\gamma=4,5,6,7$, and 9), the third column contains $r$ (the number of observations less than $\gamma$ ), the fourth column contains the ML estimate of $\alpha$, the fifth column contains the MSE of the ML estimate of $\alpha$, the sixth column contains the proportion estimate of $\alpha$, and the seventh column contains the MSE of the proportion estimate of $\alpha$. The estimates of the parameter $\beta$ from DW(II) $(1.8,0.1)$ for different $\mathrm{n}$ and the corresponding MSEs are given in Table 4.1e which constitutes of the same contents of Table $4.1 \mathrm{~d}$ after replacing $\alpha$ by $\beta$. 
4.2 For $(\alpha=1.9, \beta=0.05, d=14)$

The pmf of DW(II) $(1.9,0.05)$ is given by Table $4.2 \mathrm{a}$ and the bar chart given by Figure 4.2.

Table 4.2a. The pmf of DW(II) $(1.9,0.05)$ distribution

\begin{tabular}{|c|c|c|c|c|c|}
\hline $\mathrm{y}$ & 1 & 2 & 3 & 4 & 5 \\
\hline $\mathrm{P}(\mathrm{y})$ & 0.095 & 0.16045 & 0.19012 & 0.18341 & 0.15004 \\
\hline $\mathrm{y}$ & 6 & 7 & 8 & 9 & 10 \\
\hline $\mathrm{P}(\mathrm{y})$ & 0.1053 & 0.06333 & 0.03232 & 0.01375 & 0.00474 \\
\hline $\mathrm{y}$ & 11 & 12 & 13 & 14 & $\Sigma$ \\
\hline $\mathrm{P}(\mathrm{y})$ & 0.00127 & 0.00024 & $2.904 \times 10^{-5}$ & $1.379 \times 10^{-6}$ & 1.00000 \\
\hline
\end{tabular}

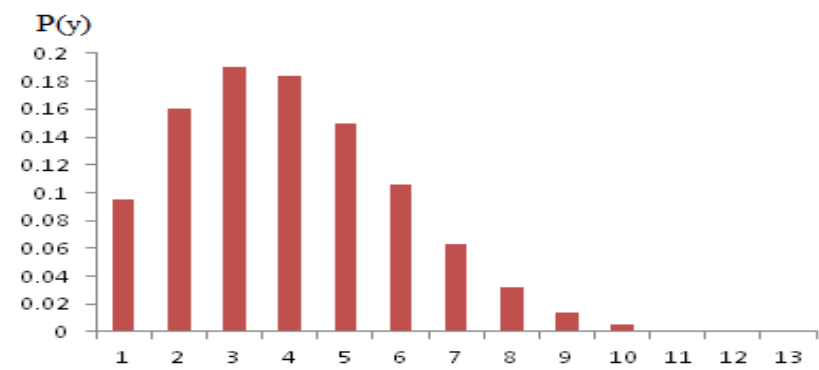

Figure 4.2 The pmf of DW(II) $(1.9,0.05)$

The first two moments and the variance of $\mathrm{DW}(\mathrm{II})(1.9,0.05)$ are respectively given by:

$\mathrm{E}(\mathrm{Y})=3.992, \mathrm{E}\left(\mathrm{Y}^{2}\right)=19.878$, and $\operatorname{Var}(\mathrm{Y})=3.942$.

The ML and the proportion estimates for the parameter $\alpha$ and $\beta$ from DW(II) $(1.9,0.05)$ for different $\gamma(\gamma=6,7,8,9$, and 14) which are found to be respectively equivalent to the values of $r(\mathrm{r}=80 \% n, 90 \% n, 95 \%$, 98\%n, and 100\%n) for each $n$ and the corresponding MSEs are given respectively in tables (4.2b) and (4.2c). Tables (4.2d) and (4.2e) represent respectively the estimates of $\alpha$ and $\beta$ from DW(II) $(1.9,0.05)$ for different $n$ and their corresponding MSEs.

4.3 For $(\alpha=1.7, \beta=0.1, d=13)$

The pmf DW(II) $(1.7,0.1)$ is given by Table 4.3a and the bar chart given by Figure 4.3.

Table 4.3a The pmf of DW(II) $(1.7,0.1)$

\begin{tabular}{|c|c|c|c|c|c|}
\hline $\mathrm{y}$ & 1 & 2 & 3 & 4 & 5 \\
\hline $\mathrm{P}(\mathrm{y})$ & 0.17 & 0.22922 & 0.22037 & 0.17067 & 0.11001 \\
\hline $\mathrm{y}$ & 6 & 7 & 8 & 9 & 10 \\
\hline $\mathrm{P}(\mathrm{y})$ & 0.05943 & 0.026755 & 0.009877 & 0.002909 & 0.000653 \\
\hline $\mathrm{y}$ & 11 & 12 & 13 & \multicolumn{2}{|c|}{$\Sigma$} \\
\hline $\mathrm{P}(\mathrm{y})$ & 0.000103 & $10^{-6} \times 9.79$ & $3.31 \times 10^{-7}$ & \multicolumn{2}{|c|}{1.00000} \\
\hline
\end{tabular}

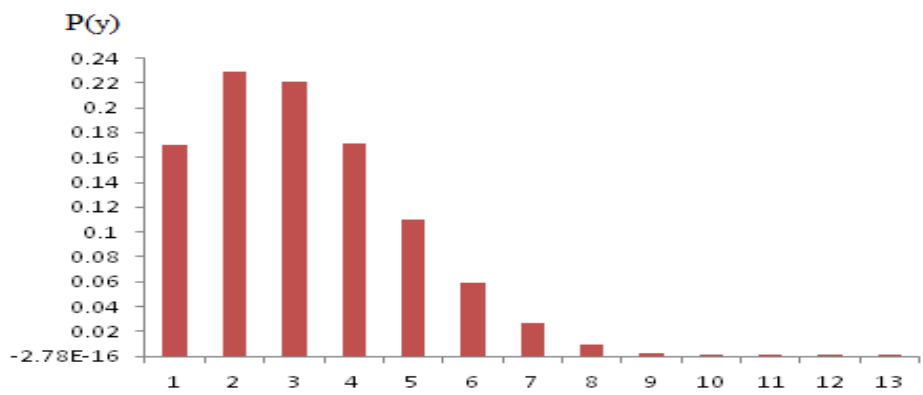

Figure 4.3 The pmf of DW(II) $(1.7,0.1)$

The first two moments and the variance of $\mathrm{DW}(\mathrm{II})(1.7,0.1)$ are respectively given by:

$$
\mathrm{E}(\mathrm{Y})=3.179, \mathrm{E}\left(\mathrm{Y}^{2}\right)=12.949 \text {, and } \operatorname{Var}(\mathrm{Y})=2.843 \text {. }
$$


The estimates of the parameters $\alpha$ and $\beta$ from $\mathrm{DW}(\mathrm{II})(1.7,0.1)$ for different $\gamma(\gamma=4,5,6,7$, and 13) which are found to be respectively equivalent to the values of $r(r=80 \% n, 90 \% n, 95 \% n, 98 \% n$, and $100 \% n)$ and the corresponding MSEs are given respectively in Table 4.3b and Table 4.3c. The estimates of $\alpha$ and $\beta$ from DW(II) (1.7, 0.1) for different $n$ and the corresponding MSEs are given respectively in Table 4.3d and Table 4.3e.

4.4 For $(\alpha=1.4, \quad \beta=0.2, d=25)$

The PMF DW(II) $(1.4,0.2)$ is given by Table $4.4 \mathrm{a}$ and the bar chart given by Figure 4.4 .

The first two moments and the variance of DW(II) $(1.4,0.2)$ are respectively given by:

$$
E(Y)=2.665, E\left(Y^{2}\right)=9.676 \text {, and } \operatorname{Var}(Y)=2.574 \text {. }
$$

The estimates of the parameters $\alpha$ and $\beta$ from $\operatorname{DW}(\mathrm{II})(1.4,0.2)$ for different $\gamma(\gamma=4,5,6,7$, and 25) which are found to be respectively equivalent to the values of $r(r=80 \% n, 90 \% n, 95 \%$, 98\%n, and $100 \% n)$ and the corresponding MSEs are given respectively in Table 4.3b and Table 4.4c. The estimates of $\alpha$ and $\beta$ from DW(II) (1.7, 0.1) for different $n$ and the corresponding MSEs are given respectively in Table $4.4 \mathrm{~d}$ and Table $4.4 \mathrm{e}$.

Table 4.4a. The pmf of DW(II) $(1.4,0.2)$

\begin{tabular}{|c|c|c|c|c|c|c|}
\hline $\mathrm{y}$ & 1 & 2 & 3 & 4 & 5 & \\
\hline $\mathrm{P}(\mathrm{y})$ & 0.28 & 0.26601 & 0.19727 & 0.12515 & 0.070129 & \\
\hline $\mathrm{y}$ & 6 & 7 & 8 & 9 & 10 & \\
\hline $\mathrm{P}(\mathrm{y})$ & 0.03523 & 0.01599 & 0.00658 & 0.00246 & 0.00084 & \\
\hline $\mathrm{y}$ & 11 & 12 & 13 & 14 & 15 & \\
\hline $\mathrm{P}(\mathrm{y})$ & 0.00026 & $7.18 \times 10^{-5}$ & $1.81 \times 10^{-5}$ & $4.07 \times 10^{-6}$ & $8.18 \times 10^{-7}$ & \\
\hline $\mathrm{y}$ & 16 & 17 & 18 & 19 & 20 & \\
\hline $\mathrm{P}(\mathrm{y})$ & $1.45 \times 10^{-7}$ & $2.25 \times 10^{-8}$ & $3.00 \times 10^{-9}$ & $3.37 \times 10^{-10}$ & $3.13 \times 10^{-11}$ & \\
\hline $\bar{y}$ & 21 & 22 & 23 & 24 & 25 & $\overline{\Sigma \Sigma}$ \\
\hline $\mathrm{P}(\mathrm{y})$ & $2.3 \times 10^{-12}$ & $1.25 \times 10^{-13}$ & $4.58 \times 10^{-15}$ & $8.67 \times 10^{-17}$ & $1.54 \times 10^{-19}$ & 1.00000 \\
\hline
\end{tabular}

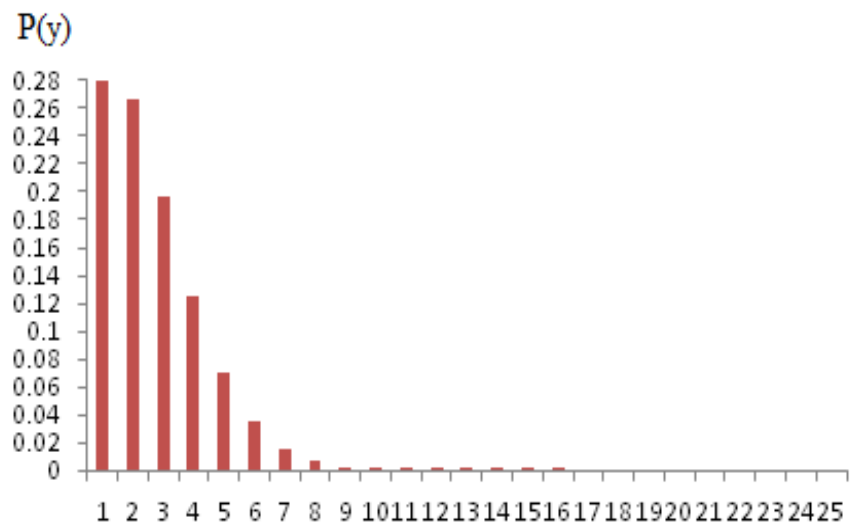

Figure 4.4 The pmf of DW(II) $(1.4,0.2)$

\section{Discussion and Conclusion}

It is observed that the simulated data in all cases don't contain all integer values in the theoretical range of the random variable, for example in the case of $\mathrm{DW}$ (II) $(1.4,0.2)$ (where, theoretically $\mathrm{Y}=1,2, \ldots, 25)$, the random variable $\mathrm{Y}$ takes only the values between 1 and 13. It takes the value 12 in twelve, seven, three, one, and one sample(s) for $n=150$, $100,50,25$, and 15 respectively, takes the value 13 only in three, two, two, one, and zero sample(s) for $n=150,100$, 50,25 , and 15 respectively, and doesn't take any of the values $14,15, \ldots$, or 25 for any sample size.

From Tables 4.1b, 4.1c, 4.1d, 4.1e, 4.2b, 4.2c, 4.2d, 4.2e, 4.3b, 4.3c, 4.3d, 4.3e, 4.4b, 4.4c, 4.4d, and 4.1e, it is observed that the ML estimates for both parameters $\alpha$ and $\beta$ are closer to the true parameter value than the proportion 
estimates, and that the MSE of the ML estimates is smaller in most cases than that of the proportion estimates. We may refer this to the number of data used in each of the estimation procedures, as the whole data set are used in the ML estimation, whereas, only a part of the data (1's and 2's only) is used in the proportion estimation, i.e. the ML estimators are more sufficient than the proportion estimators which consider only the number of 1 's and 2 's in calculating the estimates.

It seems that the estimate of the scale parameter $\beta$ is less affected by censoring than the estimate of the shape parameter $\alpha$ in most cases, especially when heavy censoring takes place.

It also seems that the ML estimates are more affected by the proportion of censored data than the proportion estimates.

Table 4.1b. Estimated $\alpha$ from DW(II) $(1.8,0.1)$ for different $\gamma$

\begin{tabular}{|c|c|c|c|c|c|}
\hline \multicolumn{6}{|c|}{$(\gamma=9) \equiv(r=100 \% n)$} \\
\hline$n$ & $r$ & $\hat{\alpha}$ & $\operatorname{MSE}(\hat{\alpha})$ & $\widetilde{\alpha}$ & $\operatorname{MSE}(\widetilde{\alpha})$ \\
\hline 15 & 15 & 1.658 & 0.2410 & $\mathbf{1 . 3 4 5}$ & 0.8835 \\
\hline 25 & 25 & 1.602 & 0.1740 & 1.296 & 0.8428 \\
\hline 50 & 50 & 1.545 & 0.1690 & 1.239 & 0.7954 \\
\hline 100 & 100 & 1.517 & 0.1780 & 1.240 & 0.7073 \\
\hline 150 & 150 & 1.513 & 0.1770 & 1.237 & 0.6909 \\
\hline \multicolumn{6}{|c|}{$(\gamma=7) \equiv(r=98 \% n)$} \\
\hline$n$ & $r$ & $\hat{\alpha}$ & $\operatorname{MSE}(\hat{\alpha})$ & $\breve{\alpha}$ & $\operatorname{MSE}(\breve{\alpha})$ \\
\hline 15 & 15 & 1.658 & 0.2410 & $\mathbf{1 . 3 4 5}$ & 0.8835 \\
\hline 25 & 25 & 1.602 & 0.1740 & 1.296 & 0.8428 \\
\hline 50 & 49 & 1.796 & 0.0680 & 1.253 & 0.7632 \\
\hline 100 & 98 & 1.853 & 0.0470 & 1.253 & 0.6775 \\
\hline 150 & 147 & 1.893 & 0.0490 & 1.250 & 0.6612 \\
\hline \multicolumn{6}{|c|}{$(\gamma=6) \equiv(r=95 \% n)$} \\
\hline$n$ & $r$ & $\hat{\alpha}$ & $\operatorname{MSE}(\hat{\alpha})$ & $\bar{\alpha}$ & $\operatorname{MSE}(\breve{\alpha})$ \\
\hline 15 & 15 & 1.658 & 0.2410 & $\mathbf{1 . 3 4 5}$ & 0.8835 \\
\hline 25 & 24 & 1.940 & 0.2610 & $\mathbf{1 . 3 2 5}$ & 0.7746 \\
\hline 50 & 48 & 1.959 & 0.1410 & 1.267 & 0.7309 \\
\hline 100 & 95 & 2.086 & 0.2320 & 1.274 & 0.6310 \\
\hline 150 & 143 & 2.058 & 0.2080 & 1.268 & 0.6209 \\
\hline \multicolumn{6}{|c|}{$(\gamma \quad=5) \equiv(r=90 \% n)$} \\
\hline$n$ & $r$ & $\hat{\alpha}$ & $\operatorname{MSE}(\hat{\alpha})$ & $\bar{\alpha}$ & $\operatorname{MSE}(\breve{\alpha})$ \\
\hline 15 & 14 & 2.336 & 0.9965 & 1.394 & 0.7797 \\
\hline 25 & 23 & 2.216 & 0.9330 & 1.358 & 0.7022 \\
\hline 50 & 45 & 2.300 & 0.6790 & $\mathbf{1 . 3 1 4}$ & 0.6285 \\
\hline 100 & 90 & 2.145 & 0.6210 & 1.313 & 0.5498 \\
\hline 150 & 135 & 1.007 & 1.2680 & 1.372 & 0.4222 \\
\hline \multicolumn{6}{|c|}{$(\gamma=4) \equiv(r=80 \% n)$} \\
\hline$n$ & $r$ & $\hat{\alpha}$ & $\operatorname{MSE}(\hat{\alpha})$ & $\bar{\alpha}$ & $\operatorname{MSE}(\check{\alpha})$ \\
\hline 15 & 12 & 2.990 & 0.8967 & 1.525 & 0.5510 \\
\hline 25 & 20 & 2.836 & 0.8725 & 1.485 & 0.4668 \\
\hline 50 & 40 & 2.943 & 0.6350 & 1.415 & 0.4414 \\
\hline 100 & 80 & 1.234 & 1.1490 & 1.410 & 0.3728 \\
\hline 150 & 120 & 1.000 & 1.2800 & 1.406 & 0.3595 \\
\hline
\end{tabular}

Note. $\hat{\alpha}=$ ML estimate for $\alpha, \breve{\alpha}=$ Proportion estimate for $\alpha$. 
Table 4.1c. Estimated $\beta$ from DW(II) $(1.8,0.1)$ for different $\gamma$

\begin{tabular}{|c|c|c|c|c|c|}
\hline \multicolumn{6}{|c|}{$(\gamma=9) \equiv(r=100 \% n)$} \\
\hline$n$ & $r$ & $\hat{\beta}$ & $\operatorname{MSE}(\hat{\beta})$ & $\bar{\beta}$ & $\operatorname{MSE}(\widetilde{\beta})$ \\
\hline 15 & 15 & 0.192 & 0.0300 & 0.489 & 0.9582 \\
\hline 25 & 25 & 0.189 & 0.0240 & 0.382 & 0.4836 \\
\hline 50 & 50 & 0.191 & 0.0190 & 0.292 & 0.0986 \\
\hline 100 & 100 & 0.190 & 0.0180 & 0.265 & 0.0628 \\
\hline 150 & 150 & 0.189 & 0.0170 & 0.260 & 0.0574 \\
\hline \multicolumn{6}{|c|}{$(\gamma=7) \equiv(r=98 \% n)$} \\
\hline$n$ & $r$ & $\hat{\beta}$ & $\operatorname{MSE}(\hat{\beta})$ & $\bar{\beta}$ & $\operatorname{MSE}(\widetilde{\beta})$ \\
\hline 15 & 15 & 0.192 & 0.0300 & 0.489 & 0.9582 \\
\hline 25 & 25 & 0.189 & 0.0240 & 0.382 & 0.4836 \\
\hline 50 & 49 & 0.094 & 0.0150 & 0.293 & 0.0984 \\
\hline 100 & 98 & 0.063 & 0.0030 & 0.267 & 0.0640 \\
\hline 150 & 147 & 0.047 & 0.0060 & 0.262 & 0.0586 \\
\hline \multicolumn{6}{|c|}{$(\gamma=6) \equiv(r=95 \% n)$} \\
\hline$n$ & $r$ & $\hat{\beta}$ & $\operatorname{MSE}(\hat{\beta})$ & $\beta$ & $\operatorname{MSE}(\beta)$ \\
\hline 15 & 15 & 0.192 & 0.0300 & 0.489 & 0.9582 \\
\hline 25 & 24 & 0.095 & 0.0040 & 0.360 & 0.3033 \\
\hline 50 & 48 & 0.062 & 0.0040 & 0.294 & 0.0983 \\
\hline 100 & 95 & 0.030 & 0.0090 & 0.270 & 0.0657 \\
\hline 150 & 143 & 0.024 & 0.0120 & 0.265 & 0.0604 \\
\hline \multicolumn{6}{|c|}{$(\gamma=5) \equiv(r=90 \% n)$} \\
\hline$n$ & $r$ & $\hat{\beta}$ & $\operatorname{MSE}(\hat{\beta})$ & $\beta$ & $\operatorname{MSE}(\beta)$ \\
\hline 15 & 14 & 0.025 & 0.0059 & 0.409 & 0.4491 \\
\hline 25 & 23 & 0.064 & 0.0047 & 0.347 & 0.2305 \\
\hline 50 & 45 & 0.030 & 0.0100 & 0.297 & 0.0979 \\
\hline 100 & 90 & 0.027 & 0.0110 & 0.274 & 0.0684 \\
\hline 150 & 135 & 0.078 & 0.0010 & 0.237 & 0.0418 \\
\hline \multicolumn{6}{|c|}{$(\gamma=4) \equiv(r=80 \% n)$} \\
\hline $\mathrm{n}$ & $\mathrm{r}$ & $\hat{\beta}$ & $\operatorname{MSE}(\hat{\beta})$ & $\bar{\beta}$ & $\operatorname{MSE}(\bar{\beta})$ \\
\hline 15 & 12 & 0.135 & 0.0064 & 0.344 & 0.2050 \\
\hline 25 & 20 & 0.159 & 0.0072 & 0.323 & 0.1457 \\
\hline 50 & 40 & 0.018 & 0.8240 & 0.301 & 0.0970 \\
\hline 100 & 80 & 0.068 & 0.0030 & 0.284 & 0.0743 \\
\hline 150 & 120 & 0.074 & 0.0010 & 0.281 & 0.0704 \\
\hline
\end{tabular}

Note. $\hat{\beta}=$ ML estimate for $\beta, \widetilde{\beta}=$ Proportion estimate for $\beta$.

Table 4.1d. Estimated $\alpha$ from DW(II) $(1.8,0.1)$ for different $n$

\begin{tabular}{|c|c|c|c|c|c|c|}
\hline \multicolumn{7}{|c|}{$(n=150)$} \\
\hline$\%$ & $\gamma$ & $r$ & $\hat{\alpha}$ & $\operatorname{MSE}(\hat{\alpha})$ & $\bar{\alpha}$ & $\operatorname{MSE}(\breve{\alpha})$ \\
\hline $80 \%$ & 4 & 120 & 1.000 & 1.2800 & 1.406 & 0.3595 \\
\hline $90 \%$ & 5 & 135 & 1.007 & 1.2680 & 1.372 & 0.4222 \\
\hline $95 \%$ & 6 & 143 & 2.058 & 0.2080 & 1.268 & 0.6209 \\
\hline $98 \%$ & 7 & 147 & 1.893 & 0.0490 & 1.250 & 0.6612 \\
\hline $100 \%$ & 9 & 150 & 1.513 & 0.1770 & 1.237 & 0.6909 \\
\hline \multicolumn{7}{|c|}{$(n=100)$} \\
\hline$\%$ & $\gamma$ & $r$ & $\hat{\alpha}$ & $\operatorname{MSE}(\hat{\alpha})$ & $\widetilde{\alpha}$ & $\operatorname{MSE}(\breve{\alpha})$ \\
\hline $80 \%$ & 4 & 80 & 1.234 & 1.1490 & 1.410 & 0.3728 \\
\hline $90 \%$ & 5 & 90 & 2.145 & 0.6210 & 1.313 & 0.5489 \\
\hline $95 \%$ & 6 & 95 & 2.086 & 0.2320 & 1.274 & 0.6310 \\
\hline $98 \%$ & 7 & 98 & 1.853 & 0.0470 & 1.253 & 0.6775 \\
\hline $100 \%$ & 9 & 100 & 1.517 & 0.1780 & 1.240 & 0.7073 \\
\hline
\end{tabular}




\begin{tabular}{|c|c|c|c|c|c|c|}
\hline \multicolumn{7}{|c|}{$(n=50)$} \\
\hline$\%$ & $\gamma$ & $r$ & $\hat{\alpha}$ & $\operatorname{MSE}(\hat{\alpha})$ & $\breve{\alpha}$ & $\operatorname{MSE}(\breve{\alpha})$ \\
\hline $80 \%$ & 4 & 40 & 2.943 & 0.6350 & 1.415 & 0.4414 \\
\hline $90 \%$ & 5 & 45 & 2.300 & 0.6790 & 1.314 & 0.6285 \\
\hline $95 \%$ & 6 & 48 & 1.959 & 0.1410 & 1.267 & 0.7309 \\
\hline $98 \%$ & 7 & 49 & 1.796 & 0.0680 & 1.253 & 0.7632 \\
\hline $100 \%$ & 9 & 50 & 1.545 & 0.1690 & 1.239 & 0.7954 \\
\hline \multicolumn{7}{|c|}{$(n=25)$} \\
\hline$\%$ & $\gamma$ & $r$ & $\hat{\alpha}$ & $\operatorname{MSE}(\hat{\alpha})$ & $\bar{\alpha}$ & $\operatorname{MSE}(\breve{\alpha})$ \\
\hline $80 \%$ & 4 & 20 & 2.836 & 0.8725 & 1.485 & 0.4668 \\
\hline $90 \%$ & 5 & 23 & 2.216 & 0.9330 & 1.358 & 0.7022 \\
\hline $95 \%$ & 6 & 24 & 1.940 & 0.2610 & 1.325 & 0.7746 \\
\hline $100 \%$ & 9 & 25 & 1.602 & 0.1740 & 1.296 & 0.8428 \\
\hline \multicolumn{7}{|c|}{$(n=15)$} \\
\hline$\%$ & $\gamma$ & $r$ & $\hat{\alpha}$ & $\operatorname{MSE}(\hat{\alpha})$ & $\widetilde{\alpha}$ & $\operatorname{MSE}(\breve{\alpha})$ \\
\hline $80 \%$ & 4 & 12 & 2.990 & 0.8967 & 1.525 & 0.5510 \\
\hline $90 \%$ & 5 & 14 & 2.336 & 0.9965 & 1.394 & 0.7797 \\
\hline $100 \%$ & 9 & 15 & 1.658 & 0.2410 & 1.345 & 0.8835 \\
\hline
\end{tabular}

Note. $\hat{\alpha}=$ ML estimate for $\alpha, \breve{\alpha}=$ Proportion estimate for $\alpha$.

Table 4.1e. Estimated $\beta$ from DW(II) $(1.8,0.1)$ for different $n$

\begin{tabular}{|c|c|c|c|c|c|c|}
\hline \multicolumn{7}{|c|}{$(n=150)$} \\
\hline$\%$ & $\gamma$ & $r$ & $\hat{\beta}$ & $\operatorname{MSE}(\hat{\beta})$ & $\bar{\beta}$ & $\operatorname{MSE}(\widetilde{\beta})$ \\
\hline $80 \%$ & 4 & 120 & 0.074 & 0.0013 & 0.281 & 0.0704 \\
\hline $90 \%$ & 5 & 135 & 0.078 & 0.0010 & 0.237 & 0.0418 \\
\hline $95 \%$ & 6 & 143 & 0.024 & 0.0120 & 0.265 & 0.0604 \\
\hline $98 \%$ & 7 & 147 & 0.047 & 0.0058 & 0.262 & 0.0586 \\
\hline $100 \%$ & 9 & 150 & 0.189 & 0.0170 & 0.260 & 0.0574 \\
\hline \multicolumn{7}{|c|}{$(n=100)$} \\
\hline$\%$ & $\gamma$ & $r$ & $\hat{\beta}$ & $\operatorname{MSE}(\hat{\beta})$ & $\bar{\beta}$ & $\operatorname{MSE}(\widetilde{\beta})$ \\
\hline $80 \%$ & 4 & 80 & 0.068 & 0.0025 & 0.284 & 0.0743 \\
\hline $90 \%$ & 5 & 90 & 0.027 & 0.0110 & 0.274 & 0.0684 \\
\hline $95 \%$ & 6 & 95 & 0.030 & 0.0935 & 0.270 & 0.0657 \\
\hline $98 \%$ & 7 & 98 & 0.063 & 0.0031 & 0.267 & 0.0640 \\
\hline $100 \%$ & 9 & 100 & 0.190 & 0.0180 & 0.265 & 0.0628 \\
\hline \multicolumn{7}{|c|}{$(n=50)$} \\
\hline$\%$ & $\gamma$ & $r$ & $\hat{\beta}$ & $\operatorname{MSE}(\hat{\beta})$ & $\bar{\beta}$ & $\operatorname{MSE}(\breve{\beta})$ \\
\hline $80 \%$ & 4 & 40 & 0.018 & 0.8240 & 0.301 & 0.0970 \\
\hline $90 \%$ & 5 & 45 & 0.030 & 0.0100 & 0.297 & 0.0979 \\
\hline $95 \%$ & 6 & 48 & 0.062 & 0.0040 & 0.294 & 0.0983 \\
\hline $98 \%$ & 7 & 49 & 0.094 & 0.0150 & 0.293 & 0.0984 \\
\hline $100 \%$ & 9 & 50 & 0.885 & 0.0190 & 0.292 & 0.0986 \\
\hline \multicolumn{7}{|c|}{$(n=25)$} \\
\hline$\%$ & $\gamma$ & $r$ & $\hat{\beta}$ & $\operatorname{MSE}(\hat{\beta})$ & $\beta$ & $\operatorname{MSE}(\beta)$ \\
\hline $80 \%$ & 4 & 20 & 0.159 & 0.0072 & 0.323 & 0.1457 \\
\hline $90 \%$ & 5 & 23 & 0.064 & 0.0047 & 0.347 & 0.2305 \\
\hline $95 \%$ & 6 & 24 & 0.095 & 0.0035 & 0.360 & 0.3033 \\
\hline $100 \%$ & 9 & 25 & 0.189 & 0.0240 & 0.382 & 0.4836 \\
\hline \multicolumn{7}{|c|}{$(n=15)$} \\
\hline$\%$ & $\gamma$ & $r$ & $\hat{\beta}$ & $\operatorname{MSE}(\hat{\beta})$ & $\beta$ & $\operatorname{MSE}(\beta)$ \\
\hline $80 \%$ & 4 & 12 & 0.135 & 0.0064 & 0.344 & 0.2050 \\
\hline $90 \%$ & 5 & 14 & 0.025 & 0.0059 & 0.409 & 0.4491 \\
\hline $100 \%$ & 9 & 15 & 0.192 & 0.0300 & 0.489 & 0.9582 \\
\hline
\end{tabular}

Note. $\hat{\beta}=$ ML estimate for $\beta, \tilde{\beta}=$ Proportion estimate for $\beta$. 
Table 4.2b. Estimated $\alpha$ from DW(II) $(1.9,0.05)$ for different $\gamma$

\begin{tabular}{|c|c|c|c|c|c|}
\hline \multicolumn{6}{|c|}{$(\gamma \quad=14) \equiv(r=100 \% n)$} \\
\hline$n$ & $r$ & $\hat{\alpha}$ & $\operatorname{MSE}(\hat{\alpha})$ & $\breve{\alpha}$ & $\operatorname{MSE}(\breve{\alpha})$ \\
\hline 15 & 15 & 1.771 & 0.2030 & 1.329 & 1.7901 \\
\hline 25 & 25 & 1.744 & 0.1440 & 1.555 & 0.9735 \\
\hline 50 & 50 & 1.690 & 0.1310 & 1.496 & 0.6553 \\
\hline 100 & 100 & 1.662 & 0.1320 & 1.394 & 0.6465 \\
\hline 150 & 150 & 1.658 & 0.1300 & 1.410 & 0.5708 \\
\hline \multicolumn{6}{|c|}{$(\gamma=9) \equiv(r=98 \% n)$} \\
\hline$n$ & $r$ & $\hat{\alpha}$ & $\operatorname{MSE}(\hat{\alpha})$ & $\breve{\alpha}$ & $\operatorname{MSE}(\breve{\alpha})$ \\
\hline 15 & 15 & 1.771 & 0.2030 & 1.329 & 1.7901 \\
\hline 25 & 25 & 1.744 & 0.1440 & 1.555 & 0.9735 \\
\hline 50 & 49 & 1.902 & 0.0620 & 1.502 & 0.6439 \\
\hline 100 & 98 & 1.926 & 0.0330 & 1.401 & 0.6326 \\
\hline 150 & 147 & 1.976 & 0.0390 & 1.416 & 0.5586 \\
\hline \multicolumn{6}{|c|}{$(\gamma=8) \equiv(r=95 \% n)$} \\
\hline$n$ & $r$ & $\hat{\alpha}$ & $\operatorname{MSE}(\hat{\alpha})$ & $\breve{\alpha}$ & $\operatorname{MSE}(\breve{\alpha})$ \\
\hline 15 & 15 & 1.771 & 0.2030 & 1.329 & 1.7901 \\
\hline 25 & 24 & 2.014 & 0.1790 & 1.567 & 0.9507 \\
\hline 50 & 48 & 2.010 & 0.0970 & 1.508 & 0.6324 \\
\hline 100 & 95 & 2.005 & 0.1050 & 1.410 & 0.6130 \\
\hline 150 & 143 & 2.100 & 0.1410 & 1.425 & 0.5411 \\
\hline \multicolumn{6}{|c|}{$(\gamma \quad=7) \equiv(r=90 \% n)$} \\
\hline$n$ & $r$ & $\hat{\alpha}$ & $\operatorname{MSE}(\hat{\alpha})$ & $\breve{\alpha}$ & $\operatorname{MSE}(\breve{\alpha})$ \\
\hline 15 & 14 & 2.121 & 0.5050 & 1.354 & 1.7127 \\
\hline 25 & 23 & 2.195 & 0.4130 & 1.581 & 0.9258 \\
\hline 50 & 45 & 2.267 & 0.4360 & 1.529 & 0.5945 \\
\hline 100 & 90 & 2.279 & 0.4070 & 1.428 & 0.5766 \\
\hline 150 & 135 & 2.014 & 0.4506 & 1.444 & 0.5045 \\
\hline \multicolumn{6}{|c|}{$(\gamma \quad=6) \equiv(r=80 \% n)$} \\
\hline$n$ & $r$ & $\hat{\alpha}$ & $\operatorname{MSE}(\hat{\alpha})$ & $\widetilde{\alpha}$ & $\operatorname{MSE}(\widetilde{\alpha})$ \\
\hline 15 & 12 & 2.379 & 1.9989 & 1.420 & 1.5227 \\
\hline 25 & 20 & 2.677 & 1.9410 & 1.631 & 0.8415 \\
\hline 50 & 40 & 2.632 & 1.3700 & 1.571 & 0.5232 \\
\hline 100 & 80 & 1.044 & 1.5420 & 1.471 & 0.4942 \\
\hline 150 & 120 & 1.000 & 1.6200 & 1.486 & 0.4273 \\
\hline
\end{tabular}

Note. $\hat{\alpha}=$ ML estimate for $\alpha, \breve{\alpha}=$ Proportion estimate for $\alpha$.

Table 4.2c. Estimated $\beta$ from DW(II) $(1.9,0.05)$ for different $\gamma$

\begin{tabular}{|c|c|c|c|c|c|}
\hline \multicolumn{6}{|c|}{$(\gamma=14) \equiv(r=100 \% n)$} \\
\hline$n$ & $r$ & $\hat{\beta}$ & $\operatorname{MSE}(\hat{\beta})$ & $\beta$ & $\operatorname{MSE}(\beta)$ \\
\hline 15 & 15 & 0.099 & 0.0098 & 0.131 & 0.1756 \\
\hline 25 & 25 & 0.094 & 0.0068 & 0.171 & 0.0602 \\
\hline 50 & 50 & 0.093 & 0.0044 & 0.137 & 0.0222 \\
\hline 100 & 100 & 0.092 & 0.0043 & $\mathbf{0 . 1 3 7}$ & 0.0200 \\
\hline 150 & 150 & 0.092 & 0.0040 & 0.128 & 0.0139 \\
\hline \multicolumn{6}{|c|}{$(\gamma=9) \equiv(r=98 \% n)$} \\
\hline$n$ & $r$ & $\hat{\beta}$ & $\operatorname{MSE}(\hat{\beta})$ & $\breve{\beta}$ & $\operatorname{MSE}(\breve{\beta})$ \\
\hline 15 & 15 & 0.099 & 0.0098 & 0.131 & 0.1756 \\
\hline 25 & 25 & 0.094 & 0.0068 & 0.171 & 0.0602 \\
\hline 50 & 49 & 0.043 & 0.0046 & 0.139 & 0.0229 \\
\hline 100 & 98 & 0.028 & 0.0010 & 0.139 & 0.0207 \\
\hline 150 & 147 & 0.020 & 0.0018 & 0.130 & 0.0146 \\
\hline
\end{tabular}




\begin{tabular}{|c|c|c|c|c|c|}
\hline \multicolumn{6}{|c|}{$(\gamma=8) \equiv(r=95 \% n)$} \\
\hline$n$ & $r$ & $\hat{\beta}$ & $\operatorname{MSE}(\hat{\beta})$ & $\widetilde{\beta}$ & $\operatorname{MSE}(\widetilde{\beta})$ \\
\hline 15 & 15 & 0.099 & 0.0098 & 0.131 & 0.1756 \\
\hline 25 & 24 & 0.044 & 0.0011 & 0.173 & 0.0604 \\
\hline 50 & 48 & 0.028 & 0.0011 & 0.141 & 0.0237 \\
\hline 100 & 95 & 0.015 & 0.0024 & 0.142 & 0.0219 \\
\hline 150 & 143 & 0.010 & 0.0032 & 0.132 & 0.0154 \\
\hline \multicolumn{6}{|c|}{$(\gamma=7) \equiv(r=90 \% n)$} \\
\hline$n$ & $r$ & $\hat{\beta}$ & $\operatorname{MSE}(\hat{\beta})$ & $\bar{\beta}$ & $\operatorname{MSE}(\widetilde{\beta})$ \\
\hline 15 & 14 & 0.025 & 0.0019 & 0.115 & 0.1736 \\
\hline 25 & 23 & 0.029 & 0.0014 & 0.171 & 0.0602 \\
\hline 50 & 45 & 0.013 & 0.0027 & 0.147 & 0.0262 \\
\hline 100 & 90 & 0.007 & 0.0035 & 0.148 & 0.0241 \\
\hline 150 & 135 & 0.042 & 0.0002 & 0.138 & 0.0175 \\
\hline \multicolumn{6}{|c|}{$(\gamma=6) \equiv(r=80 \% n)$} \\
\hline$n$ & $r$ & $\hat{\beta}$ & $\operatorname{MSE}(\hat{\beta})$ & $\bar{\beta}$ & $\operatorname{MSE}(\widetilde{\beta})$ \\
\hline 15 & 12 & 0.125 & 0.0188 & 0.066 & 0.2379 \\
\hline 25 & 20 & 0.015 & 0.0027 & 0.184 & 0.0632 \\
\hline 50 & 40 & 0.007 & 0.0037 & 0.158 & 0.0310 \\
\hline 100 & 80 & 0.040 & 0.0024 & 0.159 & 0.0290 \\
\hline 150 & 120 & 0.041 & 0.0002 & 0.150 & 0.0222 \\
\hline
\end{tabular}

Note. $\hat{\beta}=$ ML estimate for $\beta, \widetilde{\beta}=$ Proportion estimate for $\beta$.

Table 4.2d. Estimated $\alpha$ from DW(II) $(1.9,0.05)$ for different $n$

\begin{tabular}{|c|c|c|c|c|c|c|}
\hline \multicolumn{7}{|c|}{$(n=150)$} \\
\hline$\%$ & $\gamma$ & $r$ & $\hat{\alpha}$ & $\operatorname{MSE}(\hat{\alpha})$ & $\bar{\alpha}$ & $\operatorname{MSE}(\widetilde{\alpha})$ \\
\hline $80 \%$ & 6 & 120 & 1.000 & 1.6200 & 1.486 & 0.4273 \\
\hline $90 \%$ & 7 & 135 & 2.014 & 0.4506 & 1.444 & 0.5045 \\
\hline $95 \%$ & 8 & 143 & 2.100 & 0.1410 & 1.425 & 0.5411 \\
\hline $98 \%$ & 9 & 147 & 1.976 & 0.0390 & 1.416 & 0.5586 \\
\hline $100 \%$ & 14 & 150 & 1.658 & 0.1300 & 1.410 & 0.5708 \\
\hline \multicolumn{7}{|c|}{$(n=100)$} \\
\hline$\%$ & $\gamma$ & $r$ & $\hat{\alpha}$ & $\operatorname{MSE}(\hat{\alpha})$ & $\breve{\alpha}$ & $\operatorname{MSE}(\breve{\alpha})$ \\
\hline $80 \%$ & 6 & 80 & 1.044 & 1.5420 & 1.471 & 0.4942 \\
\hline $90 \%$ & 7 & 90 & 2.279 & 0.4070 & 1.428 & 0.5766 \\
\hline $95 \%$ & 8 & 95 & 2.005 & 0.1050 & 1.410 & 0.6130 \\
\hline $98 \%$ & 9 & 98 & 1.926 & 0.0330 & 1.401 & 0.6326 \\
\hline $100 \%$ & 14 & 100 & 1.662 & 0.1320 & 1.394 & 0.6465 \\
\hline \multicolumn{7}{|c|}{$(n=50)$} \\
\hline$\%$ & $\gamma$ & $r$ & $\hat{\alpha}$ & $\operatorname{MSE}(\hat{\alpha})$ & $\widetilde{\alpha}$ & $\operatorname{MSE}(\breve{\alpha})$ \\
\hline $80 \%$ & 6 & 40 & 2.632 & 1.3700 & 1.571 & 0.5232 \\
\hline $90 \%$ & 7 & 45 & 2.267 & 0.4360 & 1.529 & 0.5945 \\
\hline $95 \%$ & 8 & 48 & 2.010 & 0.0970 & 1.508 & 0.6324 \\
\hline $98 \%$ & 9 & 49 & 1.902 & 0.0620 & 1.502 & 0.6439 \\
\hline $100 \%$ & 14 & 50 & 1.690 & 0.1310 & 1.496 & 0.6553 \\
\hline \multicolumn{7}{|c|}{$(n=25)$} \\
\hline$\%$ & $\gamma$ & $r$ & $\hat{\alpha}$ & $\operatorname{MSE}(\hat{\alpha})$ & $\bar{\alpha}$ & $\operatorname{MSE}(\widetilde{\alpha})$ \\
\hline $80 \%$ & 6 & 20 & 2.677 & 1.9410 & 1.631 & 0.8415 \\
\hline $90 \%$ & 7 & 23 & 2.195 & 0.4130 & 1.581 & 0.9258 \\
\hline $95 \%$ & 8 & 24 & 2.014 & 0.1790 & 1.567 & 0.9507 \\
\hline $100 \%$ & 14 & 25 & 1.744 & 0.1440 & 1.555 & 0.9735 \\
\hline \multicolumn{7}{|c|}{$(n=15)$} \\
\hline$\%$ & $\gamma$ & $r$ & $\hat{\alpha}$ & $\operatorname{MSE}(\hat{\alpha})$ & $\bar{\alpha}$ & $\operatorname{MSE}(\breve{\alpha})$ \\
\hline $80 \%$ & 6 & 12 & 2.379 & 1.9989 & 1.420 & 1.5227 \\
\hline $90 \%$ & 7 & 14 & 2.121 & 0.5050 & 1.354 & 1.7127 \\
\hline $100 \%$ & 14 & 15 & 1.771 & 0.2030 & 1.329 & 1.7901 \\
\hline
\end{tabular}

Note. $\hat{\alpha}=$ ML estimate for $\alpha, \breve{\alpha}=$ Proportion estimate for $\alpha$. 
Table 4.2e. Estimated $\beta$ from DW(II) $(1.9,0.05)$ for different $n$

\begin{tabular}{|c|c|c|c|c|c|c|}
\hline \multicolumn{7}{|c|}{$(n=150)$} \\
\hline$\%$ & $\gamma$ & $r$ & $\hat{\beta}$ & $\operatorname{MSE}(\hat{\beta})$ & $\widetilde{\beta}$ & $\operatorname{MSE}(\widetilde{\beta})$ \\
\hline $80 \%$ & 6 & 120 & 0.041 & 0.0002 & 0.150 & 0.0222 \\
\hline $90 \%$ & 7 & 135 & 0.042 & 0.0002 & 0.138 & 0.0175 \\
\hline $95 \%$ & 8 & 143 & 0.010 & 0.0032 & 0.132 & 0.0154 \\
\hline $98 \%$ & 9 & 147 & 0.020 & 0.0018 & 0.130 & 0.0146 \\
\hline $100 \%$ & 14 & 150 & 0.092 & 0.0040 & 0.128 & 0.0139 \\
\hline \multicolumn{7}{|c|}{$(n=100)$} \\
\hline$\%$ & $\gamma$ & $r$ & $\hat{\beta}$ & $\operatorname{MSE}(\hat{\beta})$ & $\bar{\beta}$ & $\operatorname{MSE}(\bar{\beta})$ \\
\hline $80 \%$ & 6 & 80 & 0.040 & 0.0024 & 0.137 & 0.0200 \\
\hline $90 \%$ & 7 & 90 & 0.007 & 0.0035 & 0.139 & 0.0207 \\
\hline $95 \%$ & 8 & 95 & 0.015 & 0.0024 & 0.142 & 0.0219 \\
\hline $98 \%$ & 9 & 98 & 0.028 & 0.0010 & 0.148 & 0.0241 \\
\hline $100 \%$ & 14 & 100 & 0.093 & 0.0043 & 0.159 & 0.0290 \\
\hline \multicolumn{7}{|c|}{$(n=50)$} \\
\hline$\%$ & $\gamma$ & $r$ & $\hat{\beta}$ & $\operatorname{MSE}(\hat{\beta})$ & $\bar{\beta}$ & $\operatorname{MSE}(\widetilde{\beta})$ \\
\hline $80 \%$ & 6 & 40 & 0.007 & 0.0037 & 0.158 & 0.0310 \\
\hline $90 \%$ & 7 & 45 & 0.013 & 0.0027 & 0.147 & 0.0262 \\
\hline $95 \%$ & 8 & 48 & 0.028 & 0.0011 & 0.141 & 0.0237 \\
\hline $98 \%$ & 9 & 49 & 0.043 & 0.0046 & 0.139 & 0.0229 \\
\hline $100 \%$ & 14 & 50 & 0.092 & 0.0044 & 0.137 & 0.0222 \\
\hline \multicolumn{7}{|c|}{$(n=25)$} \\
\hline$\%$ & $\gamma$ & $r$ & $\hat{\beta}$ & $\operatorname{MSE}(\hat{\beta})$ & $\breve{\beta}$ & $\operatorname{MSE}(\breve{\beta})$ \\
\hline $80 \%$ & 6 & 20 & 0.015 & 0.0027 & 0.184 & 0.0632 \\
\hline $90 \%$ & 7 & 23 & 0.029 & 0.0014 & 0.175 & 0.0608 \\
\hline $95 \%$ & 8 & 24 & 0.044 & 0.0011 & 0.173 & 0.0604 \\
\hline $100 \%$ & 14 & 25 & 0.094 & 0.0068 & 0.171 & 0.0602 \\
\hline \multicolumn{7}{|c|}{$(n=15)$} \\
\hline$\%$ & $\gamma$ & $r$ & $\hat{\beta}$ & $\operatorname{MSE}(\hat{\beta})$ & $\breve{\beta}$ & $\operatorname{MSE}(\breve{\beta})$ \\
\hline $80 \%$ & 6 & 12 & 0.125 & 0.0188 & 0.066 & 0.2379 \\
\hline $90 \%$ & 7 & 13 & 0.025 & 0.0019 & 0.115 & 0.1736 \\
\hline $100 \%$ & 14 & 15 & 0.099 & 0.0098 & 0.131 & 0.1756 \\
\hline
\end{tabular}

Note. $\hat{\beta}=$ ML estimate for $\beta, \bar{\beta}=$ Proportion estimate for $\beta$

Table 4.3b Estimated $\alpha$ from DW(II) $(1.7,0.1)$ for different $\gamma$

\begin{tabular}{|c|c|c|c|c|c|}
\hline \multicolumn{7}{|c|}{$(\gamma=13) \equiv(r=100 \% n)$} \\
\hline$n$ & $r$ & $\hat{\alpha}$ & $\operatorname{MSE}(\hat{\alpha})$ & $\breve{\alpha}$ & $\operatorname{MSE}(\breve{\alpha})$ \\
\hline 15 & 15 & $\mathbf{1 . 7 7 9}$ & 0.2230 & $\mathbf{1 . 5 1 4}$ & 0.7090 \\
\hline 25 & 25 & $\mathbf{1 . 7 3 8}$ & 0.1000 & $\mathbf{1 . 4 9 9}$ & 0.3754 \\
\hline 50 & 50 & $\mathbf{1 . 7 1 6}$ & 0.0500 & $\mathbf{1 . 3 8 1}$ & 0.3273 \\
\hline 100 & 100 & $\mathbf{1 . 4 4 9}$ & 0.1430 & $\mathbf{1 . 2 4 0}$ & 0.5169 \\
\hline 150 & 150 & $\mathbf{1 . 4 4 5}$ & 0.1420 & $\mathbf{1 . 2 3 3}$ & 0.4910 \\
\hline \multicolumn{7}{|c|}{$(\gamma=7) \equiv(r=98 \% n)$} \\
\hline$n$ & $r$ & $\hat{\alpha}$ & $\operatorname{MSE}(\hat{\alpha})$ & $\breve{\alpha}$ & $\operatorname{MSE}(\breve{\alpha})$ \\
\hline 15 & 15 & $\mathbf{1 . 7 7 9}$ & 0.2230 & $\mathbf{1 . 5 1 4}$ & 0.7090 \\
\hline 25 & 25 & $\mathbf{1 . 7 3 8}$ & 0.1000 & $\mathbf{1 . 4 9 9}$ & 0.3754 \\
\hline 50 & 49 & $\mathbf{1 . 8 7 7}$ & 0.132 & $\mathbf{1 . 3 9 2}$ & 0.3119 \\
\hline 100 & 98 & $\mathbf{1 . 7 1 9}$ & 0.035 & $\mathbf{1 . 2 5 1}$ & 0.4956 \\
\hline 150 & 147 & $\mathbf{1 . 7 3 5}$ & 0.027 & $\mathbf{1 . 2 4 4}$ & 0.4699 \\
\hline
\end{tabular}




\begin{tabular}{|c|c|c|c|c|c|}
\hline \multicolumn{6}{|c|}{$(\gamma=6) \equiv(r=95 \% n)$} \\
\hline$n$ & $r$ & $\hat{\alpha}$ & $\operatorname{MSE}(\hat{\alpha})$ & $\breve{\alpha}$ & $\operatorname{MSE}(\breve{\alpha})$ \\
\hline 15 & 15 & 1.779 & 0.2230 & 1.514 & 0.7090 \\
\hline 25 & 24 & 1.915 & 0.2440 & 1.520 & 0.3529 \\
\hline 50 & 48 & 1.939 & 0.1920 & 1.404 & 0.2960 \\
\hline 100 & 95 & 1.925 & 0.1610 & 1.270 & 0.4613 \\
\hline 150 & 143 & 1.689 & 0.1870 & 1.260 & 0.4405 \\
\hline \multicolumn{6}{|c|}{$(\gamma \quad=5) \equiv(r=90 \% n)$} \\
\hline$n$ & $r$ & $\hat{\alpha}$ & $\operatorname{MSE}(\hat{\alpha})$ & $\breve{\alpha}$ & $\operatorname{MSE}(\breve{\alpha})$ \\
\hline 15 & 14 & 2.060 & 1.0530 & 1.554 & 0.7148 \\
\hline 25 & 23 & 2.047 & 0.4770 & 1.544 & 0.3300 \\
\hline 50 & 45 & 2.149 & 0.5570 & 1.442 & 0.2482 \\
\hline 100 & 90 & 2.218 & 0.6526 & 1.303 & 0.4033 \\
\hline 150 & 135 & 1.000 & 0.9800 & 1.296 & 0.3787 \\
\hline \multicolumn{6}{|c|}{$(\gamma \quad=4) \equiv(r=80 \% n)$} \\
\hline$n$ & $r$ & $\hat{\alpha}$ & $\operatorname{MSE}(\hat{\alpha})$ & $\breve{\alpha}$ & $\operatorname{MSE}(\breve{\alpha})$ \\
\hline 15 & 12 & 2.768 & 1.6336 & 1.660 & 0.5659 \\
\hline 25 & 20 & 2.564 & 1.2430 & 1.634 & 0.2658 \\
\hline 50 & 40 & 2.370 & 1.5970 & 1.523 & 0.1670 \\
\hline 100 & 80 & 3.167 & 4.5853 & 1.386 & 0.2781 \\
\hline 150 & 120 & 1.000 & 0.9800 & 1.379 & 0.2552 \\
\hline
\end{tabular}

Note. $\hat{\alpha}=$ ML estimate for $\alpha, \breve{\alpha}=$ Proportion estimate for $\alpha$.

Table 4.3c. Estimated $\beta$ from DW(II) $(1.7,0.1)$ for different $\gamma$

\begin{tabular}{|c|c|c|c|c|c|}
\hline \multicolumn{6}{|c|}{$(\gamma \quad=13) \equiv(r=100 \% n)$} \\
\hline$n$ & $r$ & $\hat{\beta}$ & $\operatorname{MSE}(\hat{\beta})$ & $\bar{\beta}$ & $\operatorname{MSE}(\widetilde{\beta})$ \\
\hline 15 & 15 & 0.155 & 0.0170 & 0.290 & 0.1979 \\
\hline 25 & 25 & 0.147 & 0.0110 & 0.204 & 0.0332 \\
\hline 50 & 50 & 0.141 & 0.0065 & 0.214 & 0.0342 \\
\hline 100 & 100 & 0.183 & 0.0160 & 0.242 & 0.0488 \\
\hline 150 & 150 & 0.182 & 0.0150 & 0.236 & 0.0414 \\
\hline \multicolumn{6}{|c|}{$(\gamma \quad=7) \equiv(r=98 \% n)$} \\
\hline$n$ & $r$ & $\beta$ & $\operatorname{MSE}(\hat{\beta})$ & $\bar{\beta}$ & $\operatorname{MSE}(\beta)$ \\
\hline 15 & 15 & 0.155 & 0.0170 & 0.290 & 0.1979 \\
\hline 25 & 25 & 0.147 & 0.0110 & 0.204 & 0.0332 \\
\hline 50 & 49 & 0.076 & 0.0023 & 0.216 & 0.0351 \\
\hline 100 & 98 & 0.389 & 0.0030 & 0.244 & 0.0498 \\
\hline 150 & 147 & 0.046 & 0.0060 & 0.238 & 0.0425 \\
\hline \multicolumn{6}{|c|}{$(\gamma,=6) \equiv(r=95 \% n)$} \\
\hline$n$ & $r$ & $\hat{\beta}$ & $\operatorname{MSE}(\hat{\beta})$ & $\beta$ & $\operatorname{MSE}(\beta)$ \\
\hline 15 & 15 & 0.155 & 0.0170 & 0.290 & 0.1979 \\
\hline 25 & 24 & 0.083 & 0.0033 & 0.207 & 0.0346 \\
\hline 50 & 48 & 0.054 & 0.0044 & 0.218 & 0.0360 \\
\hline 100 & 95 & 0.029 & 0.0100 & 0.248 & 0.0518 \\
\hline 150 & 143 & 0.038 & 0.0085 & 0.241 & 0.0442 \\
\hline \multicolumn{6}{|c|}{$(\gamma, \quad=5) \equiv(r=90 \% n)$} \\
\hline$n$ & $r$ & $\hat{\beta}$ & $\operatorname{MSE}(\hat{\beta})$ & $\bar{\beta}$ & $\operatorname{MSE}(\beta)$ \\
\hline 15 & 14 & 0.091 & 0.0056 & 0.280 & 0.1515 \\
\hline 25 & 23 & 0.062 & 0.0048 & 0.211 & 0.0363 \\
\hline 50 & 45 & 0.029 & 0.0100 & 0.224 & 0.0388 \\
\hline 100 & 90 & 0.018 & 0.0140 & 0.253 & 0.0546 \\
\hline 150 & 135 & 0.080 & 0.0007 & 0.247 & 0.0476 \\
\hline
\end{tabular}




\begin{tabular}{|c|c|c|c|c|c|}
\hline \multicolumn{7}{|c|}{$(\gamma=4) \equiv(r=80 \% n)$} \\
\hline$n$ & $r$ & $\hat{\beta}$ & $\operatorname{MSE}(\hat{\beta})$ & $\tilde{\beta}$ & $\operatorname{MSE}(\tilde{\beta})$ \\
\hline 15 & 12 & $\mathbf{0 . 1 1 0}$ & 0.0089 & $\mathbf{0 . 2 6 8}$ & 0.1109 \\
\hline 25 & 20 & $\mathbf{0 . 0 8 8}$ & 0.0069 & $\mathbf{0 . 2 2 3}$ & 0.0415 \\
\hline 50 & 40 & $\mathbf{0 . 0 2 7}$ & 0.0110 & $\mathbf{0 . 2 3 5}$ & 0.0440 \\
\hline 100 & 80 & $\mathbf{0 . 0 1 0}$ & 0.0162 & $\mathbf{0 . 2 6 4}$ & 0.0608 \\
\hline 150 & 120 & $\mathbf{0 . 0 7 7}$ & 0.0010 & $\mathbf{0 . 2 6 0}$ & 0.0550 \\
\hline
\end{tabular}

Note. $\hat{\beta}=$ ML estimate for $\beta, \vec{\beta}=$ Proportion estimate for $\beta$.

Table 4.3d. Estimated $\alpha$ from DW(II) $(1.7,0.1)$ for different $n$

\begin{tabular}{|c|c|c|c|c|c|c|}
\hline \multicolumn{7}{|c|}{$(n=150)$} \\
\hline$\%$ & $\gamma$ & $r$ & $\hat{\alpha}$ & $\operatorname{MSE}(\hat{\alpha})$ & $\breve{\alpha}$ & $\operatorname{MSE}(\breve{\alpha})$ \\
\hline $80 \%$ & 4 & 120 & 1.000 & 0.9800 & 1.379 & 0.2552 \\
\hline $90 \%$ & 5 & 135 & 1.000 & 0.9800 & 1.296 & 0.3787 \\
\hline $95 \%$ & 6 & 143 & 1.689 & 0.1870 & 1.260 & 0.4405 \\
\hline $98 \%$ & 7 & 147 & 1.735 & 0.0270 & 1.244 & 0.4699 \\
\hline $100 \%$ & 13 & 150 & 1.445 & 0.1420 & 1.233 & 0.4910 \\
\hline \multicolumn{7}{|c|}{$(n=100)$} \\
\hline$\%$ & $\gamma$ & $r$ & $\hat{\alpha}$ & $\operatorname{MSE}(\hat{\alpha})$ & $\widetilde{\alpha}$ & $\operatorname{MSE}(\breve{\alpha})$ \\
\hline $80 \%$ & 4 & 80 & 3.167 & 4.5853 & 1.386 & 0.2781 \\
\hline $90 \%$ & 5 & 90 & 2.218 & 0.6526 & 1.303 & 0.4033 \\
\hline $95 \%$ & 6 & 95 & 1.925 & 0.1610 & 1.270 & 0.4613 \\
\hline $98 \%$ & 7 & 98 & 1.719 & 0.0350 & 1.251 & 0.4956 \\
\hline $100 \%$ & 13 & 100 & 1.449 & 0.1430 & 1.240 & 0.5169 \\
\hline \multicolumn{7}{|c|}{$(n=50)$} \\
\hline$\%$ & $\gamma$ & $r$ & $\hat{\alpha}$ & $\operatorname{MSE}(\hat{\alpha})$ & $\bar{\alpha}$ & $\operatorname{MSE}(\breve{\alpha})$ \\
\hline $80 \%$ & 4 & 40 & 2.370 & 1.5970 & 1.523 & 0.1670 \\
\hline $90 \%$ & 5 & 45 & 2.149 & 0.5570 & 1.442 & 0.2482 \\
\hline $95 \%$ & 6 & 48 & 1.939 & 0.1920 & 1.404 & 0.2960 \\
\hline $98 \%$ & 7 & 49 & 1.877 & 0.1320 & 1.392 & 0.3119 \\
\hline $100 \%$ & 13 & 50 & 1.716 & 0.0500 & 1.381 & 0.3273 \\
\hline \multicolumn{7}{|c|}{$(n=25)$} \\
\hline$\%$ & $\gamma$ & $r$ & $\hat{\alpha}$ & $\operatorname{MSE}(\hat{\alpha})$ & $\breve{\alpha}$ & $\operatorname{MSE}(\breve{\alpha})$ \\
\hline $80 \%$ & 4 & 20 & 2.564 & 1.2430 & 1.634 & 0.2658 \\
\hline $90 \%$ & 5 & 23 & 2.047 & 0.4770 & 1.544 & 0.3300 \\
\hline $95 \%$ & 6 & 24 & 1.915 & 0.2440 & 1.520 & 0.3529 \\
\hline $100 \%$ & 13 & 25 & 1.738 & 0.1000 & 1.499 & 0.3754 \\
\hline \multicolumn{7}{|c|}{$(n=15)$} \\
\hline$\%$ & $\gamma$ & $\mathrm{r}$ & $\hat{\alpha}$ & $\operatorname{MSE}(\hat{\alpha})$ & $\widetilde{\alpha}$ & $\operatorname{MSE}(\breve{\alpha})$ \\
\hline $80 \%$ & 4 & 12 & 2.768 & 1.6336 & 1.660 & 0.5659 \\
\hline $90 \%$ & 5 & 14 & 2.060 & 1.0530 & 1.554 & 0.7148 \\
\hline $100 \%$ & 13 & 15 & 1.779 & 0.2230 & 1.514 & 0.7090 \\
\hline
\end{tabular}

Note. $\hat{\alpha}=$ ML estimate for $\alpha, \breve{\alpha}=$ Proportion estimate for $\alpha$. 
Table 4.3e. Estimated $\beta$ from DW(II) $(1.7,0.1)$ for different $n$

\begin{tabular}{|c|c|c|c|c|c|c|}
\hline \multicolumn{7}{|c|}{$(n=150)$} \\
\hline$\%$ & $\gamma$ & $r$ & $\hat{\beta}$ & $\operatorname{MSE}(\hat{\beta})$ & $\breve{\beta}$ & $\operatorname{MSE}(\widetilde{\beta})$ \\
\hline $80 \%$ & 4 & 120 & 0.077 & 0.0010 & 0.260 & 0.0550 \\
\hline $90 \%$ & 5 & 135 & 0.080 & 0.0007 & 0.247 & 0.0476 \\
\hline $95 \%$ & 6 & 143 & 0.038 & 0.0085 & 0.241 & 0.0442 \\
\hline $98 \%$ & 7 & 147 & 0.046 & 0.0060 & 0.238 & 0.0425 \\
\hline $100 \%$ & 13 & 150 & 0.182 & 0.0150 & 0.236 & 0.0414 \\
\hline \multicolumn{7}{|c|}{$(n=100)$} \\
\hline$\%$ & $\gamma$ & $r$ & $\hat{\beta}$ & $\operatorname{MSE}(\hat{\beta})$ & $\breve{\beta}$ & $\operatorname{MSE}(\widetilde{\beta})$ \\
\hline $80 \%$ & 4 & 80 & 0.0102 & 0.0162 & 0.264 & 0.0608 \\
\hline $90 \%$ & 5 & 90 & 0.068 & 0.0027 & 0.253 & 0.0546 \\
\hline $95 \%$ & 6 & 95 & 0.029 & 0.0100 & 0.248 & 0.0518 \\
\hline $98 \%$ & 7 & 98 & 0.389 & 0.0034 & 0.244 & 0.0498 \\
\hline $100 \%$ & 13 & 100 & 0.183 & 0.0160 & 0.242 & 0.0488 \\
\hline \multicolumn{7}{|c|}{$(n=50)$} \\
\hline$\%$ & $\gamma$ & $r$ & $\hat{\beta}$ & $\operatorname{MSE}(\hat{\beta})$ & $\bar{\beta}$ & $\operatorname{MSE}(\widetilde{\beta})$ \\
\hline $80 \%$ & 4 & 40 & 0.0270 & 0.0110 & 0.235 & 0.0440 \\
\hline $90 \%$ & 5 & 45 & 0.029 & 0.0100 & 0.224 & 0.0388 \\
\hline $95 \%$ & 6 & 48 & 0.054 & 0.0044 & 0.218 & 0.0360 \\
\hline $98 \%$ & 7 & 49 & 0.076 & 0.0023 & 0.216 & 0.0351 \\
\hline $100 \%$ & 13 & 50 & 0.141 & 0.0065 & 0.214 & 0.0342 \\
\hline \multicolumn{7}{|c|}{$(n=25)$} \\
\hline$\%$ & $\gamma$ & $r$ & $\hat{\beta}$ & $\operatorname{MSE}(\hat{\beta})$ & $\bar{\beta}$ & $\operatorname{MSE}(\breve{\beta})$ \\
\hline $80 \%$ & 4 & 20 & 0.088 & 0.0069 & 0.223 & 0.0415 \\
\hline $90 \%$ & 5 & 23 & 0.062 & 0.0048 & 0.211 & 0.0363 \\
\hline $95 \%$ & 6 & 24 & 0.083 & 0.0033 & 0.207 & 0.0346 \\
\hline $100 \%$ & 13 & 25 & 0.147 & 0.0110 & 0.204 & 0.0332 \\
\hline \multicolumn{7}{|c|}{$(n=15)$} \\
\hline$\%$ & $\gamma$ & $r$ & $\hat{\beta}$ & $\operatorname{MSE}(\hat{\beta})$ & $\breve{\beta}$ & $\operatorname{MSE}(\widetilde{\beta})$ \\
\hline $80 \%$ & 4 & 12 & 0.110 & 0.0089 & 0.268 & 0.1109 \\
\hline $90 \%$ & 5 & 14 & 0.091 & 0.0056 & 0.280 & 0.1515 \\
\hline $100 \%$ & 13 & 15 & 0.155 & 0.0170 & 0.290 & 0.1979 \\
\hline
\end{tabular}

Note. $\hat{\beta}=$ ML estimate for $\beta, \widetilde{\beta}=$ Proportion estimate for $\beta$.

Table 4.4b. Estimated $\alpha$ from DW(II) $(1.4,0.2)$ for different $\gamma$

\begin{tabular}{|c|c|c|c|c|c|}
\hline \multicolumn{6}{|c|}{$(\gamma \quad=25) \equiv(r=100 \% n)$} \\
\hline$n$ & $r$ & $\hat{\alpha}$ & $\operatorname{MSE}(\hat{\alpha})$ & $\breve{\alpha}$ & $\operatorname{MSE}(\breve{\alpha})$ \\
\hline 15 & 15 & 1.332 & 0.1140 & 1.280 & 0.2569 \\
\hline 25 & 25 & 1.265 & 0.0940 & $\mathbf{1 . 3 2 7}$ & 0.1567 \\
\hline 50 & 50 & 1.204 & 0.1030 & 1.148 & 0.2107 \\
\hline 100 & 100 & 1.179 & 0.1110 & 1.054 & 0.2821 \\
\hline 150 & 150 & 1.171 & 0.1150 & 1.001 & 0.3608 \\
\hline \multicolumn{6}{|c|}{$(\gamma=8) \equiv(r=98 \% n)$} \\
\hline$n$ & $r$ & $\hat{\alpha}$ & $\operatorname{MSE}(\hat{\alpha})$ & $\bar{\alpha}$ & $\operatorname{MSE}(\breve{\alpha})$ \\
\hline 15 & 15 & 1.332 & 0.1140 & 1.280 & 0.2569 \\
\hline 25 & 25 & 1.265 & 0.0940 & $\mathbf{1 . 3 2 7}$ & 0.1567 \\
\hline 50 & 49 & 1.363 & 0.0580 & 1.168 & 0.1904 \\
\hline 100 & 98 & 1.384 & 0.1330 & 1.074 & 0.2546 \\
\hline 150 & 147 & 1.328 & 0.0640 & 1.022 & 0.3283 \\
\hline \multicolumn{6}{|c|}{$(\gamma=6) \equiv(r=95 \% n)$} \\
\hline$n$ & $r$ & $\hat{\alpha}$ & $\operatorname{MSE}(\hat{\alpha})$ & $\breve{\alpha}$ & $\operatorname{MSE}(\breve{\alpha})$ \\
\hline 15 & 15 & 1.332 & 0.1140 & 1.280 & 0.2569 \\
\hline
\end{tabular}




\begin{tabular}{|c|c|c|c|c|c|}
\hline 25 & 24 & 1.403 & 0.1033 & 1.365 & 0.1431 \\
\hline 50 & 48 & 1.487 & 0.0940 & 1.209 & 0.1565 \\
\hline 100 & 95 & 1.471 & 0.2700 & 1.106 & 0.2137 \\
\hline 150 & 143 & 1.408 & 0.0510 & 1.050 & 0.1862 \\
\hline \multicolumn{6}{|c|}{$(\gamma \quad=5) \equiv(r=90 \% n)$} \\
\hline$n$ & $r$ & $\hat{\alpha}$ & $\operatorname{MSE}(\hat{\alpha})$ & $\bar{\alpha}$ & $\operatorname{MSE}(\breve{\alpha})$ \\
\hline 15 & 14 & 1.495 & 0.1233 & 1.433 & 0.2013 \\
\hline 25 & 23 & 1.473 & 0.2458 & 1.408 & 0.1353 \\
\hline 50 & 45 & 1.468 & 0.3190 & 1.256 & 0.1179 \\
\hline 100 & 90 & 1.501 & 0.2672 & 1.166 & 0.1483 \\
\hline 150 & 135 & 1.633 & 0.2080 & 1.114 & 0.2028 \\
\hline \multicolumn{6}{|c|}{$(\gamma \quad=4) \equiv(r=80 \% n)$} \\
\hline$n$ & $r$ & $\hat{\alpha}$ & $\operatorname{MSE}(\hat{\alpha})$ & $\bar{\alpha}$ & $\operatorname{MSE}(\widetilde{\alpha})$ \\
\hline 15 & 12 & 1.447 & 0.1688 & 1.541 & 0.2273 \\
\hline 25 & 20 & 1.435 & 0.2858 & 1.576 & 0.1821 \\
\hline 50 & 40 & 1.433 & 0.7600 & 1.403 & 0.0682 \\
\hline 100 & 80 & 1.573 & 0.6621 & 1.321 & 0.0477 \\
\hline 150 & 120 & 1.590 & 0.3200 & 1.270 & 0.0696 \\
\hline
\end{tabular}

Note. $\hat{\alpha}=$ ML estimate for $\alpha, \breve{\alpha}=$ Proportion estimate for $\alpha$.

Table 4.4c. Estimated $\beta$ from DW(II) $(1.4,0.2)$ for different $\gamma$

\begin{tabular}{|c|c|c|c|c|c|}
\hline \multicolumn{6}{|c|}{$(\gamma \quad=25) \equiv(r=100 \% n)$} \\
\hline$n$ & $r$ & $\hat{\beta}$ & $\operatorname{MSE}(\hat{\beta})$ & $\bar{\beta}$ & $\operatorname{MSE}(\bar{\beta})$ \\
\hline 15 & 15 & 0.307 & 0.0410 & 0.360 & 0.0964 \\
\hline 25 & 25 & 0.321 & 0.0420 & 0.311 & 0.0417 \\
\hline 50 & 50 & 0.332 & 0.0430 & 0.363 & 0.0670 \\
\hline 100 & 100 & 0.338 & 0.0420 & 0.397 & 0.0880 \\
\hline 150 & 150 & 0.339 & 0.0420 & 0.424 & 0.1164 \\
\hline \multicolumn{6}{|c|}{$(\gamma \quad=8) \equiv(r=98 \% n)$} \\
\hline$n$ & $r$ & $\bar{\beta}$ & $\operatorname{MSE}(\beta)$ & $\bar{\beta}$ & $\operatorname{MSE}(\beta)$ \\
\hline 15 & 15 & 0.307 & 0.0410 & 0.360 & 0.0964 \\
\hline 25 & 25 & 0.321 & 0.0420 & 0.311 & 0.0417 \\
\hline 50 & 49 & 0.189 & 0.0048 & 0.363 & 0.0665 \\
\hline 100 & 98 & 0.133 & 0.0110 & 0.396 & 0.0700 \\
\hline 150 & 147 & 0.119 & 0.0150 & 0.423 & 0.1141 \\
\hline \multicolumn{6}{|c|}{$(\gamma=6) \equiv(r=95 \% n)$} \\
\hline$n$ & $r$ & $\hat{\beta}$ & $\operatorname{MSE}(\hat{\beta})$ & $\bar{\beta}$ & $\operatorname{MSE}(\bar{\beta})$ \\
\hline 15 & 15 & 0.307 & 0.0410 & 0.360 & 0.0964 \\
\hline 25 & 24 & 0.231 & 0.0233 & 0.312 & 0.0407 \\
\hline 50 & 48 & 0.130 & 0.0130 & 0.363 & 0.0654 \\
\hline 100 & 95 & 0.162 & 0.0040 & 0.395 & 0.0854 \\
\hline 150 & 143 & 0.172 & 0.0020 & 0.421 & 0.1109 \\
\hline \multicolumn{6}{|c|}{$(\gamma \quad=5) \equiv(r=90 \% n)$} \\
\hline$n$ & $r$ & $\beta$ & $\operatorname{MSE}(\beta)$ & $\bar{\beta}$ & $\operatorname{MSE}(\hat{\beta})$ \\
\hline 15 & 14 & 0.258 & 0.0477 & 0.345 & 0.0707 \\
\hline 25 & 23 & 0.365 & 0.0384 & 0.312 & 0.0395 \\
\hline 50 & 45 & 0.120 & 0.0160 & 0.362 & 0.0389 \\
\hline 100 & 90 & 0.144 & 0.0047 & 0.393 & 0.0826 \\
\hline 150 & 135 & 0.168 & 0.0020 & 0.417 & 0.1047 \\
\hline \multicolumn{6}{|c|}{$(\gamma \quad=4) \equiv(r=80 \% n)$} \\
\hline$n$ & $r$ & $\beta$ & $\operatorname{MSE}(\beta)$ & $\beta$ & $\operatorname{MSE}(\beta)$ \\
\hline 15 & 12 & 0.211 & 0.0457 & 0.336 & 0.0592 \\
\hline 25 & 20 & 0.294 & 0.0445 & 0.312 & 0.0524 \\
\hline 50 & 40 & 0.136 & 0.0110 & 0.358 & 0.0584 \\
\hline 100 & 80 & 0.162 & 0.0030 & 0.386 & 0.0748 \\
\hline 150 & 120 & 0.178 & 0.0022 & 0.406 & 0.0916 \\
\hline
\end{tabular}

Note. $\hat{\beta}=$ ML estimate for $\beta, \quad \vec{\beta}=$ Proportion estimate for $\beta$. 
Table 4.4d. Estimated $\alpha$ from DW(II) $(1.4,0.2)$ for different $n$

\begin{tabular}{|c|c|c|c|c|c|c|}
\hline \multicolumn{7}{|c|}{$(n=150)$} \\
\hline$\%$ & $\gamma$ & $r$ & $\hat{\alpha}$ & $\operatorname{MSE}(\hat{\alpha})$ & $\bar{\alpha}$ & $\operatorname{MSE}(\breve{\alpha})$ \\
\hline $80 \%$ & 4 & 120 & 1.590 & 0.3200 & 1.270 & 0.0696 \\
\hline $90 \%$ & 5 & 135 & 1.633 & 0.2080 & 1.114 & 0.2028 \\
\hline $95 \%$ & 6 & 143 & 1.408 & 0.0510 & 1.050 & 0.1862 \\
\hline $98 \%$ & 8 & 147 & 1.328 & 0.0640 & 1.022 & 0.3283 \\
\hline $100 \%$ & 25 & 150 & 1.171 & 0.1150 & 1.001 & 0.3608 \\
\hline \multicolumn{7}{|c|}{$(n=100)$} \\
\hline$\%$ & $\gamma$ & $r$ & $\hat{\alpha}$ & $\operatorname{MSE}(\hat{\alpha})$ & $\breve{\alpha}$ & $\operatorname{MSE}(\breve{\alpha})$ \\
\hline $80 \%$ & 4 & 80 & 1.573 & 0.6621 & 1.321 & 0.0477 \\
\hline $90 \%$ & 5 & 90 & 1.501 & 0.2672 & 1.166 & 0.1483 \\
\hline $95 \%$ & 6 & 95 & 1.471 & 0.2700 & 1.106 & 0.2137 \\
\hline $98 \%$ & 8 & 98 & 1.384 & 0.1330 & 1.074 & 0.2546 \\
\hline $100 \%$ & 25 & 100 & 1.179 & 0.1110 & 1.054 & 0.2821 \\
\hline \multicolumn{7}{|c|}{$(n=50)$} \\
\hline$\%$ & $\gamma$ & $r$ & $\hat{\alpha}$ & $\operatorname{MSE}(\hat{\alpha})$ & $\breve{\alpha}$ & $\operatorname{MSE}(\breve{\alpha})$ \\
\hline $80 \%$ & 4 & 40 & 1.433 & 0.7600 & 1.403 & 0.0682 \\
\hline $90 \%$ & 5 & 45 & 1.468 & 0.3190 & 1.256 & 0.1179 \\
\hline $95 \%$ & 6 & 48 & 1.487 & 0.0940 & 1.209 & 0.1565 \\
\hline $98 \%$ & 8 & 49 & 1.363 & 0.0580 & 1.168 & 0.1904 \\
\hline $100 \%$ & 25 & 50 & 1.204 & 0.1030 & 1.148 & 0.2107 \\
\hline \multicolumn{7}{|c|}{$(n=25)$} \\
\hline$\%$ & $\gamma$ & $r$ & $\hat{\alpha}$ & $\operatorname{MSE}(\hat{\alpha})$ & $\breve{\alpha}$ & $\operatorname{MSE}(\breve{\alpha})$ \\
\hline $80 \%$ & 4 & 20 & 1.435 & 0.2858 & 1.576 & 0.1821 \\
\hline $90 \%$ & 5 & 23 & 1.473 & 0.2458 & 1.408 & 0.1353 \\
\hline $95 \%$ & 6 & 24 & 1.403 & 0.1033 & 1.365 & 0.1431 \\
\hline $100 \%$ & 25 & 25 & 1.265 & 0.0940 & 1.327 & 0.1567 \\
\hline \multicolumn{7}{|c|}{$(n=15)$} \\
\hline$\%$ & $\gamma$ & $r$ & $\hat{\alpha}$ & $\operatorname{MSE}(\hat{\alpha})$ & $\breve{\alpha}$ & $\operatorname{MSE}(\breve{\alpha})$ \\
\hline $80 \%$ & 4 & 12 & 1.447 & 0.1688 & 1.541 & 0.2273 \\
\hline $90 \%$ & 5 & 14 & 1.495 & 0.1233 & 1.433 & 0.2013 \\
\hline $100 \%$ & 25 & 15 & 1.332 & 0.1140 & 1.280 & 0.2569 \\
\hline
\end{tabular}

Note. $\hat{\alpha}=$ ML estimate for $\alpha, \bar{\alpha}=$ Proportion estimate for $\alpha$.

Table 4.4e. Estimated $\beta$ from DW(II) $(1.4,0.2)$ for different $n$

\begin{tabular}{|c|c|c|c|c|c|c|}
\hline \multicolumn{7}{|c|}{$(n=150)$} \\
\hline$\%$ & $\gamma$ & $r$ & $\hat{\beta}$ & $\operatorname{MSE}(\hat{\beta})$ & $\bar{\beta}$ & $\operatorname{MSE}(\beta)$ \\
\hline $80 \%$ & 4 & 120 & 0.178 & 0.0022 & 0.406 & 0.0916 \\
\hline $90 \%$ & 5 & 135 & 0.168 & 0.0020 & 0.417 & 0.1047 \\
\hline $95 \%$ & 6 & 143 & 0.172 & 0.0016 & 0.421 & 0.1109 \\
\hline $98 \%$ & 8 & 147 & 0.119 & 0.0150 & 0.423 & 0.1141 \\
\hline $100 \%$ & 25 & 150 & 0.339 & 0.0420 & 0.424 & 0.1164 \\
\hline \multicolumn{7}{|c|}{$(n=100)$} \\
\hline$\%$ & $\gamma$ & $r$ & $\beta$ & $\operatorname{MSE}(\hat{\beta})$ & $\beta$ & $\operatorname{MSE}(\bar{\beta})$ \\
\hline $80 \%$ & 4 & 80 & 0.162 & 0.0030 & 0.386 & 0.0748 \\
\hline $90 \%$ & 5 & 90 & 0.168 & 0.0021 & 0.393 & 0.0826 \\
\hline $95 \%$ & 6 & 95 & 0.162 & 0.0038 & 0.395 & 0.0854 \\
\hline $98 \%$ & 8 & 98 & 0.133 & 0.0110 & 0.396 & 0.0700 \\
\hline $100 \%$ & 25 & 100 & 0.338 & 0.0420 & 0.397 & 0.0880 \\
\hline
\end{tabular}




\begin{tabular}{|c|c|c|c|c|c|c|}
\hline \multicolumn{7}{|c|}{$(n=50)$} \\
\hline$\%$ & $\gamma$ & $r$ & $\hat{\beta}$ & $\operatorname{MSE}(\hat{\beta})$ & $\breve{\beta}$ & $\operatorname{MSE}(\breve{\beta})$ \\
\hline $80 \%$ & 4 & 40 & $\mathbf{0 . 1 3 6}$ & 0.0110 & $\mathbf{0 . 3 5 8}$ & 0.0584 \\
\hline $90 \%$ & 5 & 45 & $\mathbf{0 . 1 2 0}$ & 0.0160 & $\mathbf{0 . 3 6 2}$ & 0.0389 \\
\hline $95 \%$ & 6 & 48 & $\mathbf{0 . 1 3 0}$ & 0.0130 & $\mathbf{0 . 3 6 3}$ & 0.0654 \\
\hline $98 \%$ & 8 & 49 & $\mathbf{0 . 1 8 9}$ & 0.0048 & $\mathbf{0 . 3 6 3}$ & 0.0665 \\
\hline $100 \%$ & 25 & 50 & $\mathbf{0 . 3 3 2}$ & 0.0430 & $\mathbf{0 . 3 6 3}$ & 0.0670 \\
\hline \multicolumn{7}{|c|}{} \\
\hline
\end{tabular}

Note. $\hat{\beta}=$ ML estimate for $\beta, \quad \beta=$ Proportion estimate for $\beta$.

\section{References}

Chakraborty, S. (2015). Generating Discrete Analogues of Continuous Probability Distributions-A Survey of Methods and Constructions. Journal of Statistical Distributions and Applications, 2(6). https://doi.org/10.1186/s40488-015-0028-6

Khan, M. S. A., Khalique, A., \& Aboummoh, A. M. (1989). On Estimating Parameters in a Discrete Weibull Distribution. IEEE Transactions on Reliability, 38(3), 348-350. https://doi.org/10.1109/24.44179

Kulasecara, K. B. (1994). Approximate MLE's of the Parameters of a Discrete Weibull Distribution with Type-I Censored Data. Microelectronic Reliability, 34(7), 1185-1188. https://doi.org/10.1016/0026-2714(94)90502-9

Muiftah, M. S. A. (2018). On expressing continuous distributions via discrete distributions (Unpublished doctoral dissertation), FSSR, Cairo University, Egypt.

Nakagawa, T., \& Osaki, S. (1975). The discrete Weibull Distribution. IEEE Transactions on Reliability, R- 24(5), 300-301. https://doi.org/10.1109/TR.1975.5214915

Stein, W. E., \& Dattero, R. (1984). A New Discrete Weibull Distribution. IEEE Transactions on Reliability, R- 33(2), 196-197. https://doi.org/10.1109/TR.1984.5221777

\section{Copyrights}

Copyright for this article is retained by the author(s), with first publication rights granted to the journal.

This is an open-access article distributed under the terms and conditions of the Creative Commons Attribution license (http://creativecommons.org/licenses/by/4.0/). 